$69^{\text {th }}$ International Astronautical Congress (IAC), Bremen, Germany, 1-5 October 2018. Copyright 2018 by German Aerospace Center (DLR), Bremen, Germany. Published by the IAF, with permission and released to the IAF to publish in all forms.

IAC-18.A3.4B.2x42783

\title{
Size Matters - The Shell Lander Concept for Exploring Medium-Size Airless Bodies
}

\author{
Christian D. Grimm ${ }^{\mathrm{a} *}$, Silvio Schröder ${ }^{\mathrm{a}}$, Lars Witte ${ }^{\mathrm{a}}$, Kai Wickhusen ${ }^{\mathrm{b}}$ \\ ${ }^{a}$ German Aerospace Center (DLR), Institute of Space Systems, Robert-Hooke-Str.7, 28359 Bremen, Germany, \\ ${ }^{\mathrm{b}}$ German Aerospace Center (DLR), Institute of Planetary Research, Rutherfordstraße 2, 12489 Berlin, Germany, \\ * Corresponding Author, christian.grimm@dlr.de
}

\begin{abstract}
Once addressed as a side topic in planetary exploration, the investigation of small solar system bodies has now become one of the corner stones in the international science community in order to study the formation of the solar system and the evolution of life within. For rendezvous spacecraft, small carry-on landers have proven to be valuable assets, and to have a positive impact on the overall mission cost, by avoiding additional complexity of the main satellite and transferring the risk of close surface maneuvers entirely or at least to some extend to an independent deployable system. However, carry-on landers have been designed currently to land on very small bodies only, but medium-size class objects between diameters of $10-50 \mathrm{~km}$ are of great interest as well. In this paper we classify carry-on landers with respect to their touchdown and operational strategy, evaluate the constraints of ballistic deployments for different target bodies as well as identify the niche for using simple honeycomb impact dampers compared to optional retro-propulsion systems. Further we introduce the system design of a guided Shell Lander using a generic instrument carrier attached to a single ejectable crash-pad with stabilizing capability to protect the instrument carrier from structural damage, limit internal shock loads for sensitive payloads as well as reduce the amount of bounces on the surface. Finally, we present a mission architecture for a reference case to the Martian moon Phobos as well as provide a proof of concept based on laboratory impact tests.
\end{abstract}

Keywords: (Asteroid Landing, Payload Delivery, Impact Energy Absorption, Shell Lander, Ejectable Crash-Pad,)

\section{Introduction}

In recent years, the exploration of small solar system bodies has increased significantly. Apart from remote sensing surveys and fly-bys, especially landing and sample collection missions have dramatically increased our understanding of their physical composition as well as their mechanical properties. Small carry-on landers, like the European Rosetta lander Philae [1] which successfully landed on comet 67P/Churyumov/ Gerasimenko in November 2014, or the German/French lander MASCOT [2] on-board the Japanese Hayabusa2 mission (Fig. 1) scheduled for landing on asteroid 162173 Ryugu on October $3^{\text {rd }}, 2018$, have proven to be a valuable asset by avoiding additional complexity of the main satellite and keeping project development times and costs in manageable bounds.

Landing on small bodies is particular difficult due to the weak gravitational field and means to secure the lander to the surface have to be taken into account. However, with increasing size and density of the target the gravitational attraction on a lander increases also. Currently, non-propelled landers have been designed to land on very small bodies only, but medium-size class objects between diameters of $10-50 \mathrm{~km}$ are of great interest as well. For example, the Martian moons Phobos $(D=22 \mathrm{~km})$ and Deimos $(D=12.5 \mathrm{~km})$ as well

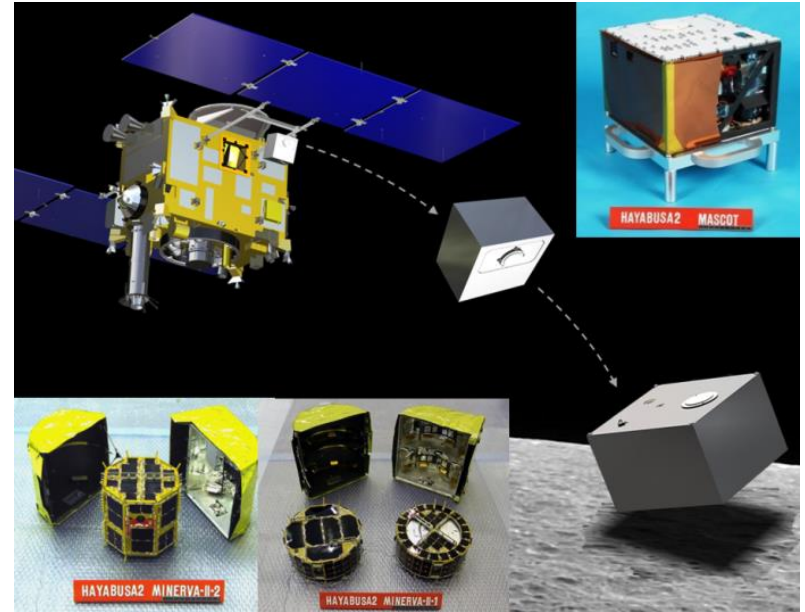

Fig. 1: Landing on Small Bodies. Example from the Hayabusa2 mission including 4 deployable landers

as many Main-belt or Jupiter Trojan asteroids have mean diameters of more than $10 \mathrm{~km}$. Rendezvous missions to those targets considering a detachable lander will have to focus on a dedicated landing support system. Depending on the capabilities of the mother spacecraft and resulting landing strategy, mainly the separation altitude defines the final landing velocity at touchdown. Higher landing velocities introduce high shock loads and can cause damage to lander subsystems 
and instruments. Reducing the need of an optional retropropulsion system, other means of cancelling the descent $\Delta v$ such as absorbing the impact energy have to be taken into careful consideration. Non-propelled landing strategies can be divided into three categories.

(i) Landing without a dedicated landing subsystem for very low touchdown velocities (e.g. MASCOT),

(ii) Landing with energy absorption to reduce the impact velocity to stay below the target's escape velocity (e.g. Philae), and

(iii) Heavy duty landing with a dedicated protection system to lower internal shock loads.

In order to enable the exploration and landing on medium-size airless bodies, this paper outlines the concept of advancing small body landers with a crushable-shell protection system to sustain higher landing velocities in the range of $1-5 \mathrm{~m} / \mathrm{s}$. Design aspects as well as possible reference missions are given in addition to results of initial crushable-shell impact tests.

\section{Application and Reference Missions}

The increased interest for small body science is seen not only by the scientific results already gained by passed missions and the expected outcome of current flight missions, but also by the continuous effort of the international scientific community to propose and to undertake missions to small bodies. Such missions can be characterized by four partially interlinked subdisciplines, (i) Solar System and Asteroid Science, (ii) Planetary Defence, (iii) Human Exploration and (iv) Resource Utilisation [3]. In recent years another subdiscipline, Mining and Exploitation of asteroids for rare materials, became also a bit more of attention. However, with the current price tag on space missions and involving time durations a justifiable concept has yet to be put forward.

Current selected missions, addressing one or more of the above topics, include the Double Asteroid Redirect Test (DART) from NASA/APL targeting to impact on the secondary of a binary asteroid (proposed launch Dec. 2020), the Lucy Jupiter Trojan multiple target rendezvous mission from NASA Goddard/SwRI (proposed launch Oct. 2021), the Psyche space probe to a metal-rich M-class asteroid by NASA/JPL/ASU (proposed launch mid 2022) and the Mars Moon eXplorer (MMX) by JAXA (proposed launch Sep. 2024) targeting the two moons of Mars, Phobos and Deimos. A European proposal to accompany the DART mission, the Asteroid Impact Monitor (AIM), was original not selected, but is currently being reinstated as Hera [4] to observe the aftereffects of the DART impact (proposed launch Oct.2023).

Typically, small body missions, be it fly-bys, orbiting spacecraft or landing systems are big space probes, whose development costs range in the hundreds of million US\$ for small-class missions up to more than a billion US\$ for high priority flagship missions. For example, several Small Body Missions have been performed by NASA within its small-class mission Discovery Program, which range between 300-500M\$ (completed missions: NEAR, Deep Impact, Stardust and Dawn; planned missions: Psyche and Lucy) [5][6]. The currently running New Frontiers medium-class mission OSIRIS-REx has a cost cap of $850 \mathrm{M} \$$ plus launch vehicle [7] and the European Rosetta mission, a cornerstone mission within ESA's Horizon 2000 Program, came with a price tag of approximately $1.5 \mathrm{~B} \$$ [8]. However, specifically the carry-on landers can avoid additional complexity of the main satellite and transferring the risk (and cost) of close surface maneuvers entirely or at least to some extend to an independent deployable system. Generally, small instrument packages and dedicated landers can scientifically enhance a main mission by either providing ground truth for the mission's orbital investigations, exploring niches on the surface too difficult or too risky to be reached for the main satellite, or adding complementary in-situ investigations with higher and long-stable resolution [9][10]. Good examples for this are the MINERVA and MASCOT landers on the Hayabusa and Hayabusa2 mission. Other concepts worth mentioning are the POGO concept from the Applied Physics Laboratory (APL) [11], the Hedgehog platform from the Jet Propulsion Laboratory (JPL) [12][13] as well as the advanced MASCOT-2 design from the German Aerospace Center (DLR) which has been proposed in the frame of the ESA AIM mission [14][15]. The advancement and further development of carry-on systems has the potential to further stimulate low cost participation in planetary exploration and increase the science return of any mission exploring these unique solar system bodies.

The aspect of lowering the overall cost of missions to main-belt and near-Earth objects, as well as the benefits to include smaller carry-on smallsats, explicitly including the addition of a lander asset, has recently been addressed also in the Small Planetary Platforms (SPP) assessment performed by ESA [16] in Nov-Dec. 2017 as well as in Jan. 2018. This study was to evaluate a possible "tool box" of technical building blocks that the community can use to develop new planetary missions consisting of a mothership spacecraft carrying a swarm of smallsats to be deployed for multi-point science observations within a cost range of up to $150 \mathrm{M} €$ $(175 \mathrm{M} \$)$. For such mission architectures, these "assets" will need to be as flexible and robust as possible to cope with varying science and mission constraints. 


\section{Classification of Small Body Landers}

Due to the fact that only a few small body landings have been attempted and even less have been successful (see Table 1), a comprehensive classification of this type of landing system is rather difficult. At the time of writing, 9 attempts have been made to put a man-made object onto the surface of a low gravity body (only 4 of these have been successful). Two missions are currently in operation including scenarios to land 3 types of carryon landers as well as 2 different types of touch-and-go manoeuvres in order take surface samples. One mission is currently in development. And out of multiple pure academic design studies 3 are being regarded as having reached an high enough technological readiness level (TRL) to be considered for an upcoming small body mission. In order to find similarities and to identify key technologies an evaluation is made based on these 18 past, present and possible future landers.

A primary parameter here is the kinetic energy at touchdown (TD), which is defined as the work done by a lander with mass $\boldsymbol{m}$ when decelerating from its incoming speed $v$ to a state of rest

$$
\mathrm{E}_{\mathrm{k}}=\frac{1}{2} \cdot \mathrm{m} \cdot \mathrm{v}^{2}
$$

The data is presented in Fig. 2 which reveals cluster in specific regimes summarized in Fig. 3. The easiest distinction of Small Body Landers (SBL) is between Orbiter Landers and Carry-on Landers. Orbiter landers are the complete probe (or final separated stage) of a rendezvous spacecraft which is intended to make physical contact with the target either during a planned mission operation or as an end of life strategy. Orbiter landers use their on-board attitude control thrusters and other reaction controls like fly wheels in order to reduce and limit the contact speed (e.g. NEAR, Fobus Grunt, Rosetta, MMX). A special case is given by touch-andgo samplers which, strictly speaking, do not actually land, but only touch the surface with some form of extended sample mechanism (e.g. Hayabusa 1+2, OSIRIS REx).

Carry-on landers do rely on an orbiter spacecraft by which they are carried to the specified target. Once arrived, they are separated in order to make the remaining journey towards the surface independently. All carry-on landers which have either been flown to date or have been seriously studied did not use or take retro-propulsion systems into account, since landing speeds were low enough that the final $\Delta \mathrm{v}$ could safely be made by impact forces only. Naturally, if for some future mission design the limit for high TD velocities will be reached a dedicated deceleration system using conventional thrusters will be the usual way forward. As it is shown in Fig. 2 and Fig. 3, carry-on landers can further be divided with respect to their operational and

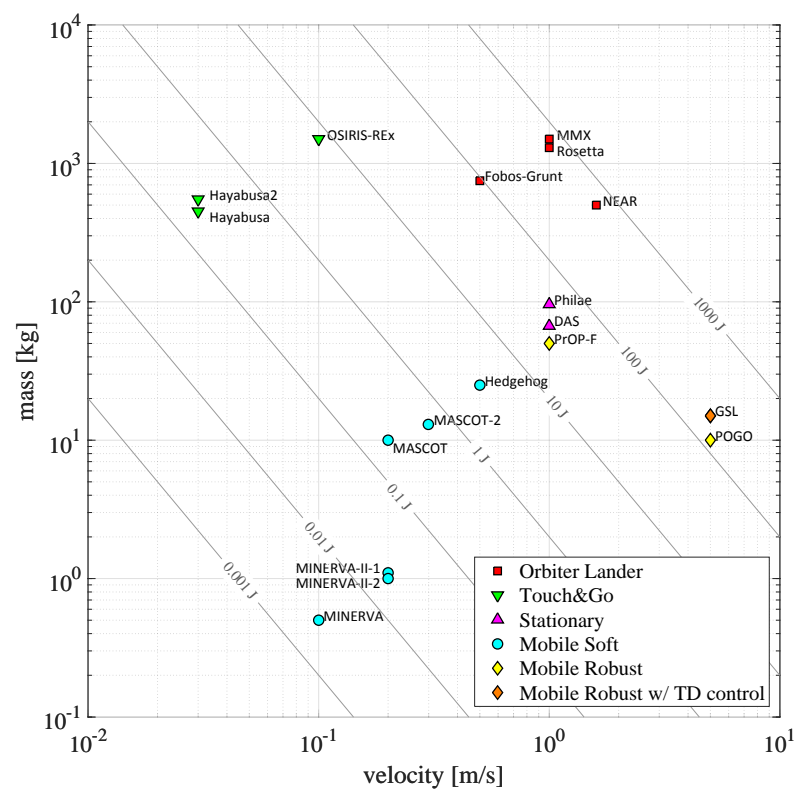

Fig. 2: Touchdown velocity-mass-diagram indicating the touchdown energy for certain types of SBL systems (underlying data from Table 1, display adapted from [26]) for low gravity bodies.

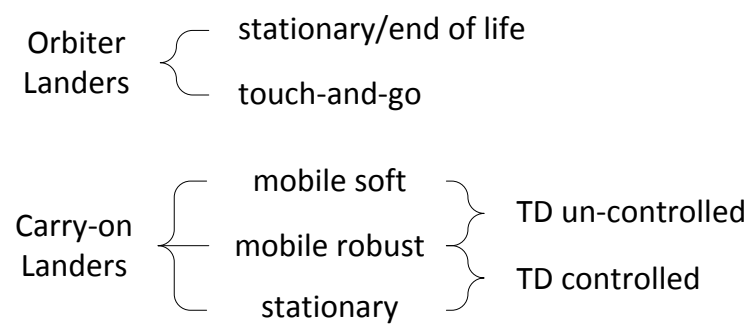

Fig. 3: Clustering of SBL systems with respect to touchdown and operational concept

touchdown strategy into mobile soft, mobile robust and stationary landers and are being either TD controlled or TD un-controlled. Mobile soft landers are usually small surface instruments without attitude control and with very low impact $\Delta \mathrm{v}$. The corresponding shock acceleration at surface contact, after a tumbling descent, is some orders of magnitude lower than the endured shock and vibration environment of a given launcher system. No structural reinforcement, impact attenuation or special landing support system is necessary. Up until now all soft landers did incorporate some form of mobility concept to either upright after landing or to move across the surface to enlarge the scientific area (e.g. MINERVA 1+2, MASCOT 1+2, Hedgehog).

A variation of mobile landers designed to survive higher landing velocities is found in mobile robust landers, where the harder landing is taken by a reinforced outer casing (e.g. PrOP-F, POGO). 
$69^{\text {th }}$ International Astronautical Congress (IAC), Bremen, Germany, 1-5 October 2018. Copyright 2018 by German Aerospace Center (DLR), Bremen, Germany. Published by the IAF, with permission and released to the IAF to publish in all forms.

Table 1: Past, present and possible future Small Body Landers

\begin{tabular}{|c|c|c|c|c|c|c|c|c|}
\hline Mission & Target & Lander & Carry-on & System Mass [kg] & Launch & Landing & TD vel $[\mathrm{m} / \mathrm{s}]$ & Ref \\
\hline Fobos 1 & \multirow{3}{*}{ Phobos } & Fobos 1 DAS & yes & \multirow{2}{*}{67} & 07.07 .88 & N/A & $<1$ & \multirow{3}{*}[17]{} \\
\hline Fobos 2 & & Fobos 2 DAS & yes & & \multirow{2}{*}{12.07 .88} & N/A & $<1$ & \\
\hline (CCCP) & & Fobos 2 PrOP-F & yes & 50 & & N/A & $<1$ & \\
\hline $\begin{array}{c}\text { NEAR } \\
\text { (NASA) }\end{array}$ & Eros & orbiter & - & 820 (wet), 500 (at TD) & 17.02 .96 & 12.02 .01 & $\sim 1.6$ & {$[17]$} \\
\hline Hayabusa & \multirow{2}{*}{ Itokawa } & orbiter & - & 510 (wet), 450 (at TD) & \multirow{2}{*}{ 19.11.05 } & 19.11 .05 & $\sim 0.03$ & \multirow{2}{*}{$\begin{array}{l}{[18} \\
{[19}\end{array}$} \\
\hline$(\mathrm{JAXA})$ & & MINERVA & yes & 0.5 & & N/A & $\sim 0.1$ & \\
\hline Rosetta & \multirow{2}{*}{ Chury-G. } & orbiter & - & 2900 (wet), 1300 (at TD) & \multirow{2}{*}{02.03 .04} & 30.09 .16 & $\sim 1$ & \multirow{2}{*}{ [1] } \\
\hline (ESA) & & Philae & yes & 96 & & 12.11 .14 & $\sim 1$ & \\
\hline $\begin{array}{c}\text { Fobos- } \\
\text { Grunt } \\
\text { (Russia) }\end{array}$ & Phobos & lander stage & - & 1820 (wet), 750 (at TD) & 08.11 .11 & N/A & $\sim 0.5$ & {$[20]$} \\
\hline \multirow{4}{*}{$\begin{array}{c}\text { Hayabusa2 } \\
\text { (JAXA) }\end{array}$} & \multirow{4}{*}{ Ryugu } & orbiter & - & 609 (wet), 550 (at TD) & \multirow{4}{*}{14.12 .14} & 10.18 & $\sim 0.03$ & \multirow{4}{*}{$\begin{array}{r}{[21]} \\
{[2]}\end{array}$} \\
\hline & & MINERVA-II-1a+b & yes & $2 \times 1.1$ & & 21.09 .18 & $\sim 0.2$ & \\
\hline & & MINERVA-II-2 & yes & 1 & & 07.19 & $\sim 0.2$ & \\
\hline & & MASCOT & yes & 10 & & 03.10 .18 & $\sim 0.2$ & \\
\hline $\begin{array}{c}\text { OSIRIS-Rex } \\
\text { (NASA) }\end{array}$ & Bennu & orbiter & - & 2110 (wet), 1500 (at TD) & 08.09 .16 & $\begin{array}{l}2019 \\
\text { (TBD) } \\
\end{array}$ & $\sim 0.1$ & $\begin{array}{c}{[7]} \\
{[22]}\end{array}$ \\
\hline $\begin{array}{l}\text { MMX } \\
\text { (JAXA) }\end{array}$ & Phobos & lander stage & - & 3400 (wet), 1500 (at TD) & 09.2024 & 2025 & $\sim 1$ & {$[23]$} \\
\hline TBD & TBD & $\begin{array}{c}\text { MASCOT-2 } \\
\text { (DLR) }\end{array}$ & yes & 13 & - & - & $<0.3$ & \\
\hline TBD & TBD & $\begin{array}{c}\text { Hedgehog } \\
(\mathrm{JPL})\end{array}$ & yes & 25 & - & - & $<0.5$ & $\begin{array}{l}{[24]} \\
{[25]}\end{array}$ \\
\hline TBD & TBD & $\begin{array}{c}\text { POGO } \\
\text { (APL) }\end{array}$ & yes & 10 & - & - & $5(\mathrm{TBC})$ & [11] \\
\hline
\end{tabular}

Stationary systems (one surface contact only) also take the impact energy into account. But in contrast to mobile robust systems, the descent is stabilized and directed (TD control) so that the surface contact is made with a defined orientation. They have to entirely absorb the impact energy and/or counteract the rebound after surface contact. Usually a combination of different subsystems is used including landing gears, internal impact dampers, hold-down thrusters and surface anchors (e.g. DAS, Philae). If this TD control principle is applied to the mobile systems we find another subcategory, mobile robust with touchdown control. Like the mobile robust systems, which have no attitude control, they incorporate some form of relocation mechanism. But since the TD is directed only the particular area which is intended to make the first physical contact with the surface needs to be equipped with a special damper or absorber system. This latter concept, however, has up until now neither been flown or studied. For this reason, this paper introduces the concept of a Guided Shell Lander (GSL). In the following section the application niche in the $\Delta \mathrm{v}$ regime is identified where impact protection by mechanical dampers and absorbers remain beneficial for a use without additionally required propulsive $\Delta \mathrm{v}$ reduction.

\subsection{Rationale and application niche for the shell lander concept}

Honeycomb material, mainly from aluminum alloys, has already a long tradition as reliable energy absorption means for impact and crash attenuation application. Its energy absorption capability comes from deliberately buckling, collapsing and plastic deformation of the hexagonal walls of the cells when loaded. The compression strength required to crush it is determined by the size and the aluminum foil gauges used to form the honeycomb cells. Crush strength and bulk density are designed, type-specific properties of such honeycomb material. The crush strength of commercially available aluminum (alloy 5052 and 5056) honeycomb samples [27] is plotted over its bulk density in Fig. 4 below. Regression curves are plotted 
additionally, showing that the crush strength is roughly - proportional to the square of the material's bulk density $\left(\sigma \propto \rho^{2}\right)$. We use this relation to make a basic assessment of the mass efficiency of honeycomb material if used as decelerator for a small landing probe.

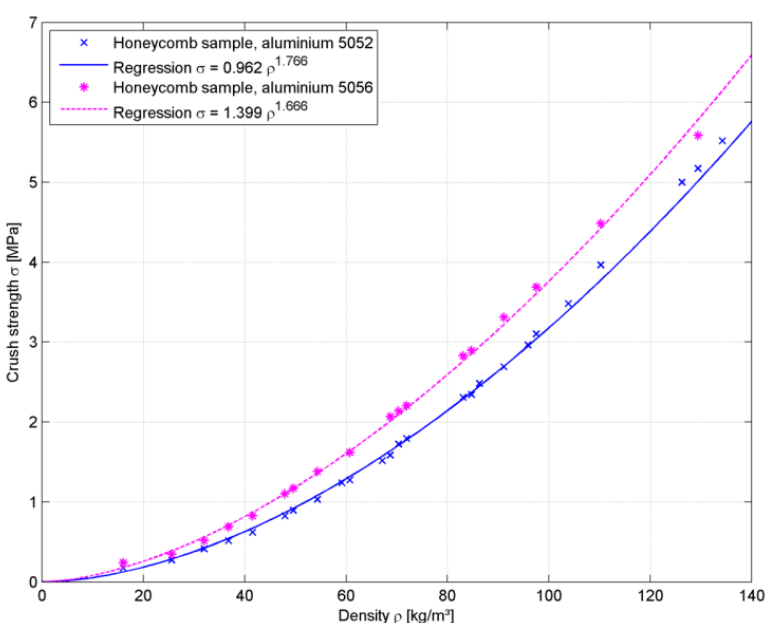

Fig. 4: Crush strength dependence as function of honeycomb bulk density. Data points of samples taken from [27]

Let $E_{k}$ be the kinetic energy at touchdown with $m_{\text {total }}$ the total mass which is the combined mass of the to-bedelivered system $m_{s y s}$ and the required crushable absorber mass $m_{c r}$. The kinetic energy shall be absorbed through plastic deformation of the shell such that $\mathrm{E}_{\mathrm{k}}=\mathrm{E}_{\text {plast }}$.

$$
\mathrm{E}_{\mathrm{k}}=\frac{1}{2} \mathrm{~m}_{\mathrm{total}} \cdot \mathrm{v}^{2}=\mathrm{F}_{\mathrm{cr}} \cdot \mathrm{s}=\sigma \cdot \underbrace{\mathrm{A} \cdot \mathrm{s}}_{:=\mathrm{V}_{\mathrm{cr}}}=\mathrm{E}_{\text {plast }}
$$

The latter is the product of the crush force $F_{c r}$ and the deformation stroke $s$. The crush force in turn is the product of the honeycomb material's crush strength $\sigma$ and the contact area $A$. For a symmetrical absorber with a constant contact area (e.g. cuboid or cylinder), the parameters $A$ and $s$ represent also the crushing volume $V_{c r}$. The crush volume can be expressed as a function of the crush strength $\sigma$ and crushable mass $m_{c r}$ using the functional relation $\sigma \approx k \cdot \rho^{2}$ as shown in Fig. 4 .

$$
\mathrm{V}_{\mathrm{cr}}=\frac{\mathrm{m}_{\mathrm{cr}}}{\rho}=\sqrt{\frac{\mathrm{k}}{\sigma}} \cdot \mathrm{m}_{\mathrm{cr}}
$$

This expression can be reformulated as the mass ratio of the required crushable mass $m_{c r}$ with regard to the system's mass $m_{s y s}$ as a function of the landing velocity or $\Delta v$ respectively.

$$
\frac{\mathrm{m}_{\mathrm{cr}}}{\mathrm{m}_{\mathrm{sys}}}=\frac{\Delta \mathrm{v}^{2}}{2 \sqrt{\mathrm{k} \sigma}-\Delta \mathrm{v}^{2}}
$$

This equation has a singularity at

$$
\Delta \mathrm{v}=\left[2 \cdot(k \cdot \sigma)^{0.5}\right]^{0.5}
$$

where the mass ratio of $m_{c r}$ to $m_{s y s}$ rises to infinity. Travelling with this $\Delta v$ a piece of honeycomb material contains more kinetic energy than can be absorbed in a deliberate, controlled manner by intended collapsing of its honeycomb cells. Obviously, the use of such material becomes extremely inefficient when approaching this limit $\Delta v$. This limit is however dependent to the respective materials crush strength.

For comparison reasons, a similar expression has been derived from the classical rocket equation using a propulsion subsystem to decelerate the landing probe.

$$
\frac{\mathrm{m}_{\text {prop }}}{\mathrm{m}_{\mathrm{sys}}}=1+\lambda-\mathrm{e}^{-\Delta \mathrm{v} /\left(\mathrm{I}_{\mathrm{sp}} \cdot \mathrm{g}_{0}\right)}
$$

In equation (6) the "decelerator mass" $m_{\text {prop }}$ is composed of the fixed propulsion subsystem mass and the $\Delta v$-dependent propellant mass. The fixed subsystem mass representing the tank, tubes and regulators and the thruster is expressed as a mass share $\lambda$. The figure $\lambda-$ assumed to be $\lambda \approx 0.1-$ is approximated using the mass budget values available from off-the-shelf cube sat propulsion hardware. For this application a cold gas system with a specific impulse of $60 \mathrm{~s}$ is assumed. An overview of such propulsion systems is given in [28] and a particular application to small body missions is studied in [29]. Although these propulsion cube sat hardware is developed primarily for orbiting elements, it is assumed here that it can be adapted in principal to deliver a shorter but higher thrust braking burn for the purpose of landing.

The resulting mass ratios expressed in equation (4) and (6) as function of landing $\Delta v$ of both crushable and propulsive means are plotted in Fig. 5. The $\Delta v$ regime with the crushable materials mass ratio being lower than its benchmark for a propulsion subsystem marks the niche where a crushable shell can offer an advantage in terms of mass efficiency. Not accounted are associated mass contributions from the respective structural or mounting support and any required guidance and control subsystem. A full trade-off must include also additionally factors such as mission design flexibility and system reliability. Due to its simplicity, in the following sections the application niche for this shell lander technology is exploited to design a highly reliable delivery system for a small asteroid landing probe. 


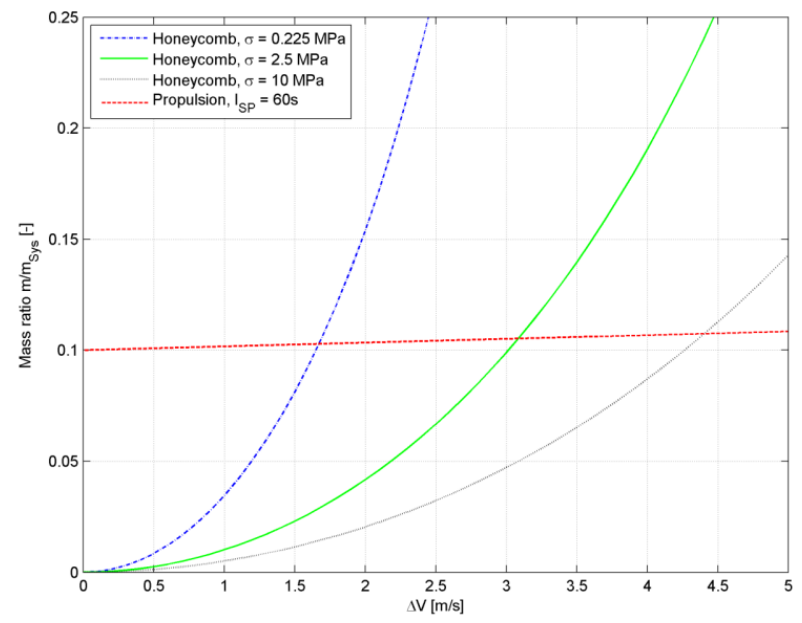

Fig. 5: Mass ratios of decelerator mass - crushable shell with different crush strength and propulsion using cold gas - shown as function of landing $\Delta v$

\section{Constraints and Limitations}

Without retro-propulsion for deceleration, the landing speed of a carry-on lander is entirely dependent by Newtons law of gravity. The higher and therefore the longer the free fall within a gravitational field the higher the impact velocity. Up to now carry-on landers have been designed to and been successfully proven for vertical touchdown velocities of $0-1 \mathrm{~m} / \mathrm{s}$. This included on the lower end soft landers without impact attenuation, and on the upper end landers with impact attenuation and hold down/anchor systems. From historical experience of other planetary landing systems (e.g. Moon and Mars) it is known to which residual vertical landing speed a system has to be decelerate in order to perform a "soft landing" that does not result in damage or destruction of the vehicle or anything on board $(\sim 5 \mathrm{~m} / \mathrm{s})[26]$.

The gravitational environment of a target body is determined by its mass defined foremost by its size but also by its bulk density which depends on the bodies material composition and its internal structure. The density is related to the object's spectral type. Estimations presented in [30] give mean bulk densities of the three main classifications $\mathrm{C}, \mathrm{S}$, and $\mathrm{M}$ type asteroids as $1.38 \pm 0.02,2.71 \pm 0.02$, and $5.32 \pm 0.07$ $\mathrm{g} / \mathrm{cm}^{3}$, respectively. Comets are less dense as they are mainly composed of water ice, dust and other frozen volatiles. In addition, they commonly have large macroporosities of $>60 \%$ forming large voids inside suggesting bulk densities of less than $1.0 \mathrm{~g} / \mathrm{cm}^{3}$, with a most likely value of $0.6 \pm 0.2 \mathrm{~g} / \mathrm{cm}^{3}$ [31]. This becomes apparent when comparing the values in Fig. 6, showing the relation of the targets size and respective surface acceleration.

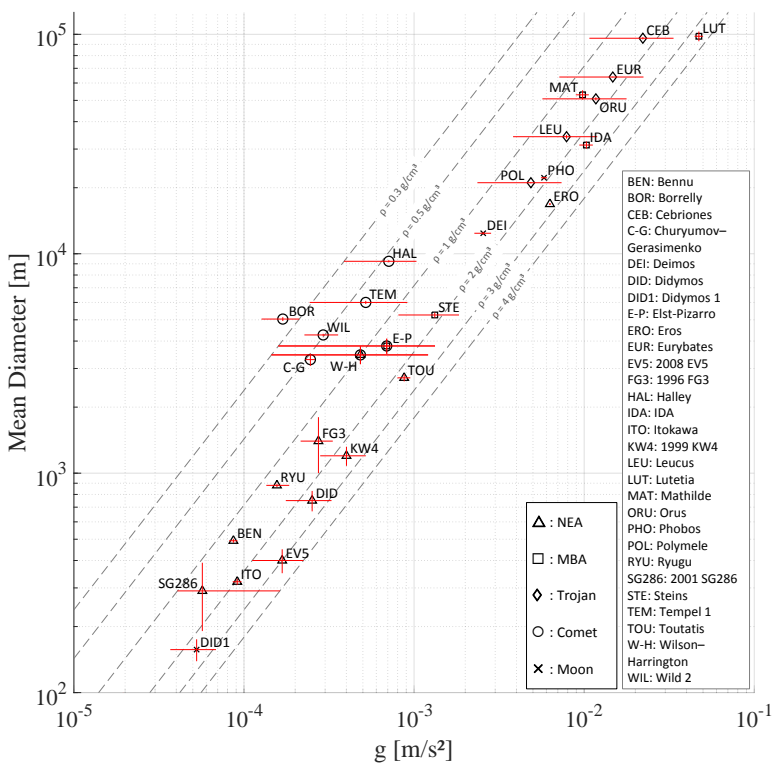

Fig. 6: Log-log plot of the gravitational attraction with respect to mean diameter and bulk density of possible target objects including error margins [32]

For example, Bennu (BEN), B-type, having a slightly lower surface acceleration than Itokawa (ITO), S-type, although being 50\% larger. Didymos (DID), Stype, having a slightly higher surface acceleration than comet Churyumov/ Gerasimenko (C-G) although being only a quarter of its size. The gravitational influence of the target is one of the primary factors determining the design of a small body lander. Depending on the gravitational potential $\boldsymbol{g}$ and the deployment altitude $\boldsymbol{h}$ estimates of the landing velocity $\boldsymbol{g}$ can be made. In a constant gravity environment this can be determined by balancing the potential energy $\boldsymbol{E}_{p}$ at release with the kinetic energy at impact $\boldsymbol{E}_{\boldsymbol{k}}$.

$$
E=E_{k}+E_{p}=\frac{1}{2} m \vartheta^{2}+m \cdot g \cdot h
$$

As the lander will not lose any mass during its descent we could simplify this to

$$
\vartheta=\sqrt{2 g h}
$$

However, if the target bodies are small or the separation altitude is much larger than the radius of the target body we have to account for varying acceleration. Since the gravitational attraction is in this case not constant. For this, we have to integrate the equation of motion which is given by Newton's law of gravity describing that the target body with mass $\boldsymbol{M}$ and the lander with mass $\boldsymbol{m}$ will attract each other with a combined force of

$$
F=\frac{G M m}{r^{2}}=m \cdot a
$$


where $\boldsymbol{G}$ is the gravitational constant and $\boldsymbol{r}$ is the distance towards the centres of gravity. As $\boldsymbol{a}$ is the acting acceleration on the lander we get the equation of motion within the gravity field towards the surface by

$$
g=-a=-\frac{d \vartheta}{d t}=-\frac{G M}{r^{2}}
$$

Multiplying with $\boldsymbol{\vartheta}$ and integrating we get the expression for the total energy of the two objects

$$
E=\frac{1}{2} \vartheta^{2}-\frac{G M}{r}
$$

where the first part is again the kinetic energy and the second part the potential energy. And as energy is always conserved the value for the energy at the moment of separation has to be the same value as the energy at the moment of impact $\left(E_{\text {sep }}=E_{\text {imp }}\right)$. Using now the values for the respective moment in time we get the amount of energy at separation with

$$
E_{\text {sep }}=\frac{1}{2} \vartheta_{0}^{2}-\frac{G M}{r_{0}}
$$

where $\boldsymbol{r}_{\boldsymbol{0}}$ is the distance from the point of separation towards the gravity centre of the target body and $v_{0}$ is the initial velocity given to the lander, which is a combination of the relative velocity of the spacecraft and the eject velocity of the separation mechanism. The energy at impact is then given respectively with

$$
E_{i m p}=\frac{1}{2} \vartheta_{i m p}{ }^{2}-\frac{G M}{r}
$$

where $\boldsymbol{r}$ is the radius of the target body and $\boldsymbol{v}_{\text {imp }}$ the terminal velocity at impact. Solving for $\boldsymbol{v}_{\boldsymbol{i m p}}$ we are left with the expression

$$
\vartheta_{i m p}=\sqrt{\vartheta_{0}{ }^{2}-2 G M\left(\frac{1}{r_{0}}-\frac{1}{r}\right)}
$$

accounting for both varying acceleration and nonvanishing initial velocity. Setting $\boldsymbol{v}_{\boldsymbol{0}}$ to be 0 and $\boldsymbol{r}_{\boldsymbol{0}}$ to be infinite we get an expression for the targets escape velocity on its surface.

$$
\vartheta_{e s c}=\sqrt{\frac{2 G M}{r}}
$$

With a target simplified as a spherical and symmetric body of mass $\boldsymbol{M}$, expressed by its volume and bulk density $\rho$

$$
M=V \cdot \rho=\frac{4}{3} \pi r^{3} \cdot \rho
$$

We can now transform equation (15) into

$$
\vartheta_{e s c}=\sqrt{\frac{8 G \pi r^{2}}{3} \cdot \rho}
$$

And respectively from equation (10) we get the magnitude of the targets gravitational potential with

$$
g_{\text {target }}=\frac{4 G \pi r}{3} \cdot \rho
$$

With this we have now all expressions to analyze the impact velocity of a lander, separated at a given altitude over a target body expressed in terms of size and density. Fig. 7 shows the estimation of impact velocity with respect to surface acceleration and deployment height for the MASCOT-1 lander on asteroid Ryugu, the Rosetta lander Philae on comet Churyumov/ Gerasimenko and a possible future carry-on lander to the Martian moon Phobos. Possible scenarios for varying deployment altitudes are given in addition to the targets respective escape velocities. According to equation (14) and the respective target size and density the velocity increase during the free fall to the surface can vary greatly between a few centimeters to meters per second. For small bodies of a few hundred meters in diameter and resulting low gravities the initial separation velocity determines to great effect the resulting touch down speed, which both are of the same order of magnitude as the escape velocity of the body. This was in fact the case for the landing of Philae, where the impact speed was slightly higher as the targets escape velocity (due to the relative high initial speed) [1]. But this was than fortunately damped both by the soft surface as well as the internal electromechanical damper system. This effect weakens as the targets grow bigger as here the velocity increase due to the higher gravitational free fall acceleration dominates. However, this means also that even at low deployment altitudes the touchdown speed would rise very quickly to a few $\mathrm{m} / \mathrm{s}$. If we take for example a separation altitude of $1 \mathrm{~km}$ on Phobos, the vertical touchdown velocity would be already $3.3 \mathrm{~m} / \mathrm{s}$. And for this regime of impact velocity one should consider some form of protection to ensure the lander itself is not fractured at impact as well as to reduce the resulting shock acceleration for the landers subsystem, specifically for sensitive instruments like cameras and other optics. 

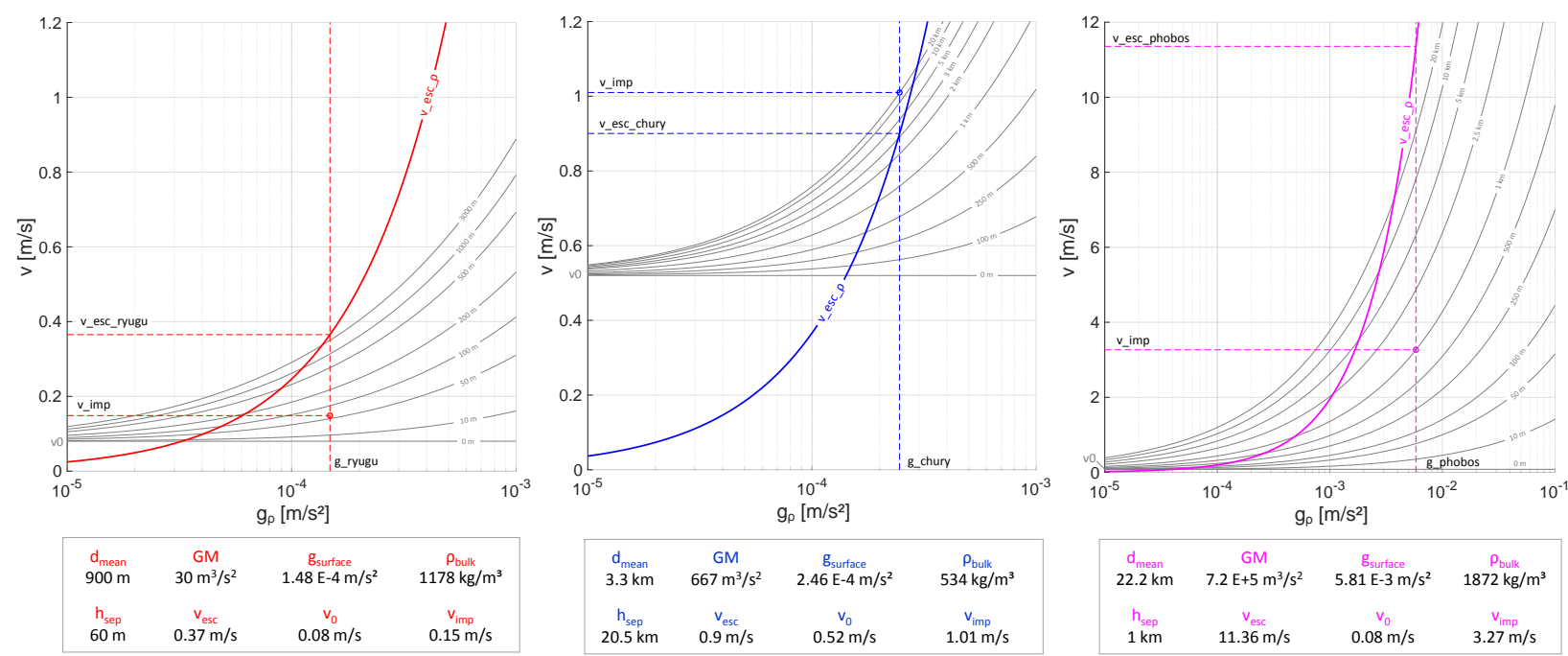

Fig. 7: Estimations of impact velocity with respect to surface acceleration and deployment height for the MASCOT-1 lander on asteroid Ryugu (left), the Philae lander on comet Churyumov/Gerasimenko (middle) and a possible future carry-on lander to the Martian moon Phobos (right).

\section{Mission Analysis and Concept of Operation}

Using the Martian moon Phobos as a reference case being both a good example for the class of a mediumsize airless body as well as being a scientifically interesting target object we present in the following a mission analysis and concept of operation for the delivery of the proposed shell lander.

With the strongly perturbing gravity of Mars and the mass of Phobos being too small to capture a satellite, it is not possible to orbit the Martian moon in the usual sense. However, orbits of a special kind - generally referred to as distant retrograde orbits, also called quasisatellite orbits (QSO) - exist and can be sufficiently stable to allow several months of operations in the vicinity of the moon. Typical QSO's are only stable for inclinations up to approximately $30^{\circ}$ relative to the equator of Phobos and at distances above $20 \mathrm{~km}$ relative to the center of the moon. In this simulation we start in a QSO at a closest distance of $25 \mathrm{~km}$ and an inclination of $0^{\circ}$. For the Lander delivery we assume a separation height of 1-3 km above the surface of Phobos. To reach this altitude the spacecraft will use its thruster and perform maneuvers which bring the spacecraft closer to the surface. In the simulation we decelerated the speed by a $\Delta v=2 \mathrm{~m} / \mathrm{s}$ to bring the spacecraft on a close fly-by course, with the closest distance to the surface < $3 \mathrm{~km}$ (see Fig. 8). During the closest approach the speed over ground is approximately $10 \mathrm{~m} / \mathrm{s}$. For lander separation a relative speed of $<1 \mathrm{~m} / \mathrm{s}$ is required. Consequently, before separation the S/C will have to decelerate to that speed over ground and release the lander. Afterwards the spacecraft has to accelerate to the previous flyby speed again and perform an additional maneuver at a distance of $25 \mathrm{~km}$ to enter

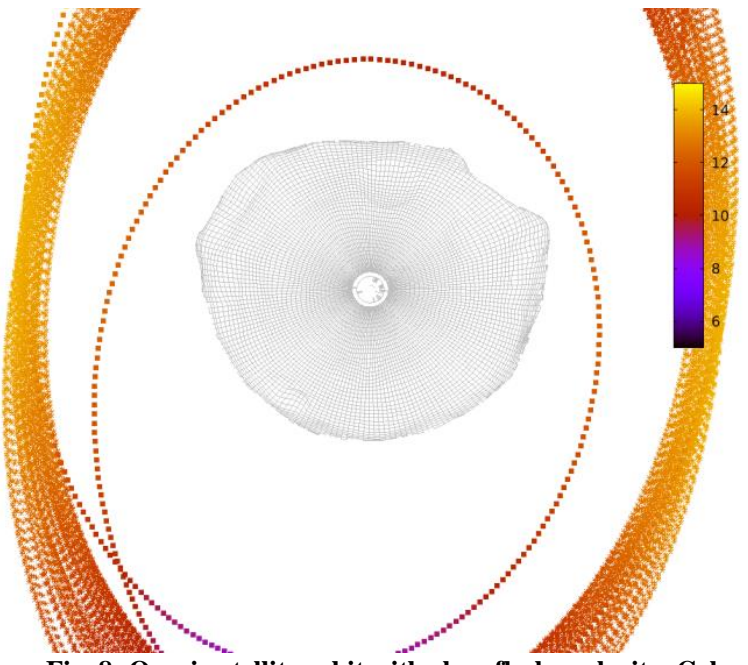

Fig. 8: Quasi-satellite orbit with close fly-by velocity. Color coding in $\mathrm{m} / \mathrm{s}$ to Phobos surface.

original QSO again. The overall delta-v demand for these 4 maneuvers is $\Delta \mathrm{v}<30 \mathrm{~m} / \mathrm{s}$ and will increase to $\Delta \mathrm{v}<60 \mathrm{~m} / \mathrm{s}$ for a delivery in the polar area. Directly after separation from the mother spacecraft, the gravity of Phobos will cause a continuously accelerated free fall. Depending on the release altitude, the impact speed and free fall time vary. At $1 \mathrm{~km}$ altitude the impact speed after 10 minutes of free falling is about $3 \mathrm{~m} / \mathrm{s}$ (Fig. 9), while at release altitude of $3 \mathrm{~km}$ the lander will fall for 20 minutes and touch the surface with an impact speed of approximately 5-6 m/s. (see also Fig. 7).

Fig. 10 shows the concept of the shell lander delivery. As mentioned above, the carrying mother spacecraft will perform a close fly-by with a relative speed above ground. 


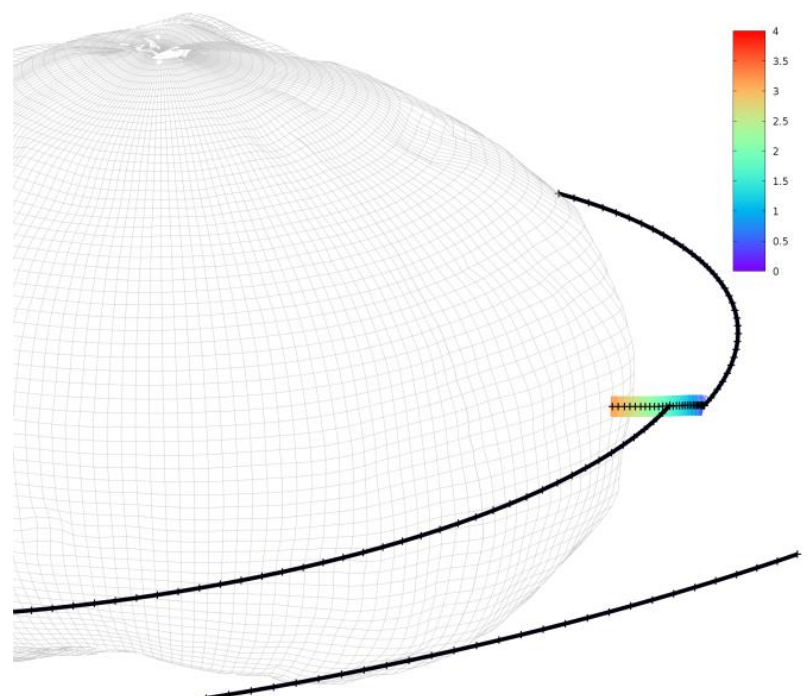

Fig. 9: Lander delivery from close flyby. Color coding in $\mathrm{m} / \mathrm{s}$ to Phobos surface. Each cross marks one minute.

The shell lander will be deployed retrograde against the normal flight path to reduce the vertical velocity component. Afterwards the shell lander will perform a ballistic free fall towards the surface (see section 4). Since the mobile instrument package has only one protective shell at its bottom side the descent has to be stabilized about the vertical axis. This can be achieved with the aid of a simple single momentum wheel (flywheel) within the shell platform. This strategy is inherited by the Rosetta Lander Philae which was also fly-wheel stabilized during its ballistic descent. Details with regard to this strategy are found in [33].
After the free fall and consequent continued acceleration due to the targets gravity, the shell lander will impact onto the surface with a defined velocity in the order of a few meters per second. The kinetic energy at the moment of impact will be absorbed by the plastic deformation of the crushable shell element (crash-pad) as well as in the best case also by the granular displacement of the regolith material. This however is not the design case of the crash-pad, since we have to assume that the lander will hit either a flat bare rock or even worse a sharp and solid boulder. The deformation of the crash-pad alone will protect the instrument package from structural damage as well as reduces the resulting shock accelerations within by one or two orders of magnitude (see section 7). This ensures on the one hand the survivability of the carried sensitive instruments and electronics and on the other limits the remaining deflected kinetic energy for the following bouncing phase which reduces the size of the landing ellipse. After the first touchdown and damped impact, the shell platform can either be released directly to free the mobile unit early (Mode-1, Fig. 10) or be kept to damp rolling in order to limit the landing area further (Mode-2). The latter is a concept already proposed by the failed PrOP-F lander which used a wire frame to limit bouncing and uncontrolled rolling over the surface [34][35][36]. The release of the shell would follow in this case after the lander comes to rest. Once freed from the shell, the mobile unit will come to rest in one location where normal surface operation can start by an up-righting or jump to another location.

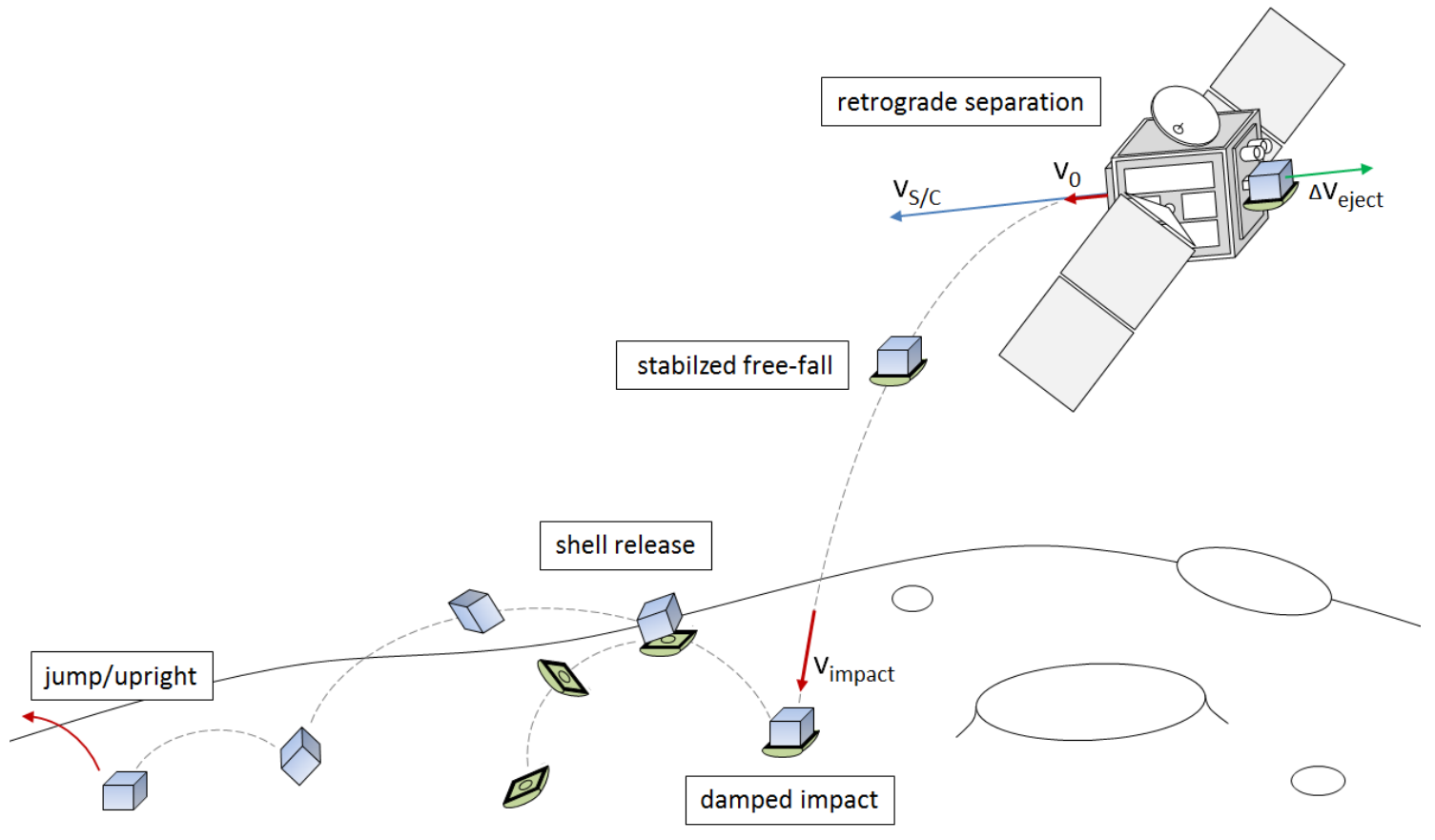

Fig. 10: Schematic view of the shell lander delivery and touchdown scenario (Mode 1) 


\section{System Design}

To obtain a reference system design we focus on the class of SBL which have recently most often been studied and reported. These are the DLR MASCOTType, the APL POGO and the JPL Hedgehog (refer to section 3 for their general classification). All have in common that they are mobile surface assets. In addition to the crushable shells primary task to safely decelerate the lander and attenuate the impact shocks we require here that it must be separated after landing to enable the mobile element an unobstructed further surface operation.

The design of the Guided Shell Lander (GSL) shown in Fig. 11 is based on a mobile instrument carrier attached to an Ejectable Crash Pad (ECAP). We present in the following a design concept using the MASCOT-2 lander [14][15] depicted in Fig. 12 as a baseline. But we would like to emphasize that the ECAP design is flexible and can be adapted to support other types of small body landers (see section 6.2).

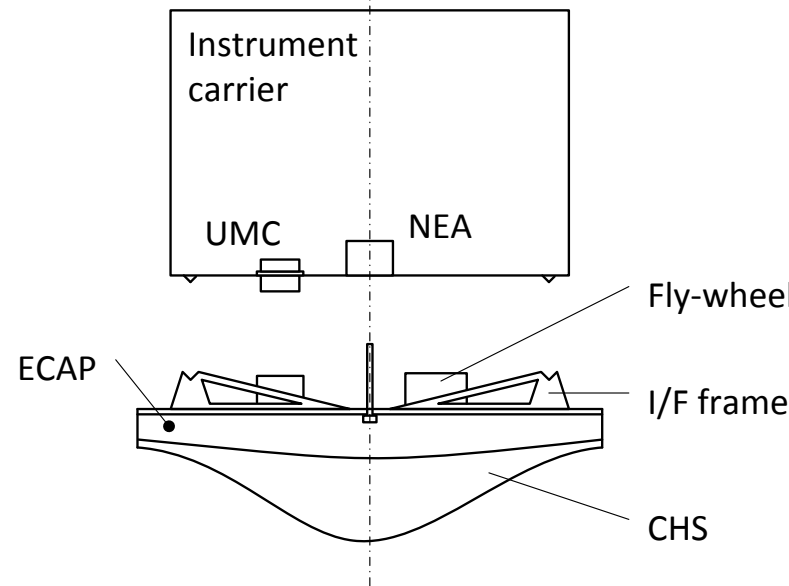

Fig. 11: Guided Shell Lander (GSL) schematic.

The main part of the ECAP system is the Crushable Half-Shell (CHS) made of a honeycomb core material glued to a high strength face sheet laminate, which is designed explicitly to the given mission requirements. On top of this shell rests an interface structure with a sandwich plate and a frame of vertical cross beams aligned in an x-pattern, which bear the loads both for the landing impact as well as for the required preload for launch. This interface structure is also the platform on which the supporting electronics are integrated, including the stabilizing flywheel, the separable umbilical connector (UMC) as well as additional sensors, such as an inertia measurement unit (IMU) or tri-axial accelerometers $(3 \mathrm{xAcc})$. This way the ECAP could be used as an instrument itself acquiring data on surface mechanical properties such as the compressive strength and effective E-modulus of the soil, as well as the damping properties between the soil and the lander. The mobile carrier is attached via 4 stand-offs transferring all remaining loads into its primary structure. The mechanical fixation as well as the release capability is ensured via a single non-explosive actuator (NEA), which needs to be allocated within the mobile unit. No additional push-off mechanism is necessary as this is provided by the release of the preload stored within the cross beams as well as the spring contacts inside the UMC. The mass breakdown can be seen in Table 2.

Table 2. Mass budget of the shell lander (MASCOT-2 baseline) including the Ejectable Crash Pad (ECAP) and an interface structure for the attachment to a mother spacecraft.

\begin{tabular}{|c|c|c|c|}
\hline Component & $\begin{array}{c}\text { Mass w/o } \\
\text { margin } \\
{[\mathrm{kg}]}\end{array}$ & $\begin{array}{c}\text { Maturity } \\
\text { margin } \\
{[\%]} \\
\end{array}$ & $\begin{array}{c}\text { Mass w/ } \\
\text { margin } \\
{[\mathrm{kg}]}\end{array}$ \\
\hline \multicolumn{4}{|c|}{ Mobile Instrument Carrier (MASCOT-2) } \\
\hline Mobility & 1,02 & 9 & 1,11 \\
\hline GNC & 0,44 & 36 & 0,60 \\
\hline On-Board Computer & 0,56 & 11 & 0,62 \\
\hline Communication & 0,88 & 18 & 1,04 \\
\hline Power Supply & 3,43 & 11 & 3,81 \\
\hline Structure incl. Solar P. & 2,23 & 20 & 2,67 \\
\hline Thermal Control & 0,22 & 5 & 0,23 \\
\hline System Harness & 1,33 & 8 & 1,44 \\
\hline Payloads & 2,01 & 15 & 2,31 \\
\hline Total & 12,12 & & 13,83 \\
\hline \multicolumn{4}{|c|}{ Spacecraft Interface and Deployment } \\
\hline Structure & 0,45 & 18 & 0,53 \\
\hline Harness & 0,16 & 13 & 0,18 \\
\hline Release and Push-off & 0,50 & 30 & 0,65 \\
\hline Calibration Targets & 0,12 & 17 & 0,14 \\
\hline Total & 1,23 & & 1,50 \\
\hline \multicolumn{4}{|c|}{ Landing Sub-System (ECAP) } \\
\hline CHS & 0,500 & 10 & 0,55 \\
\hline I/F Plate & 0,250 & 10 & 0,28 \\
\hline I/F Frame & 0,250 & 20 & 0,30 \\
\hline NEA incl. bolt & 0,080 & 5 & 0,08 \\
\hline UMC incl. Harness & 0,065 & 5 & 0,07 \\
\hline Fly-wheel & 0,150 & 30 & 0,20 \\
\hline $\begin{array}{l}\text { Optional Sensors } \\
\text { (e.g. IMU or 3xAcc) }\end{array}$ & 0,020 & 5 & 0,02 \\
\hline Total & 1,32 & & 1,49 \\
\hline Grand Total & 14,67 & & 16,82 \\
\hline
\end{tabular}

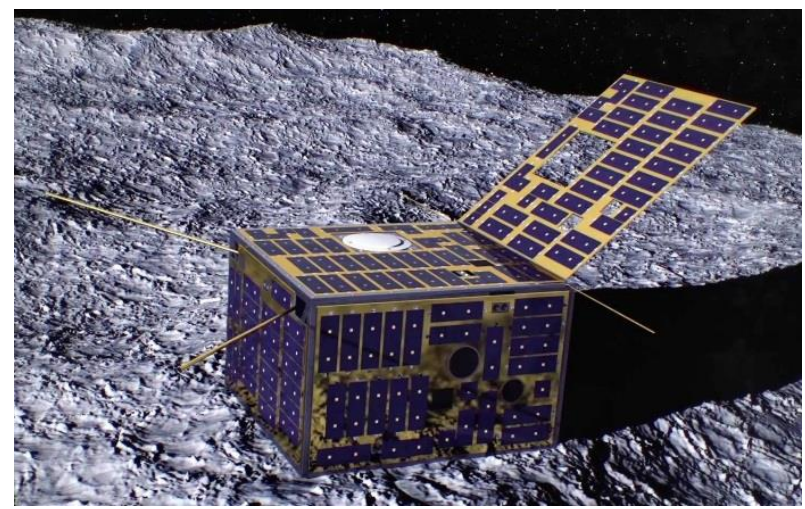

Fig. 12: Artist impression of the MASCOT-2 lander which was developed by DLR and proposed for the ESA AIM mission (image credit: ESA). 


\subsection{Design Concept}

Earlier design concepts included a full-shell enclosure (Fig. 13). This was based on the previous MASCOT-1 design where no attitude control was foreseen and the lander would make a tumbling descent followed by multiple bounces across the surface. Once at rest, the shell would unfold in order to provide unhindered visibility of internal instruments.
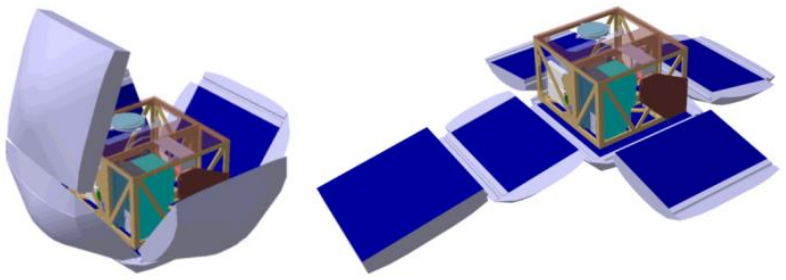

Fig. 13: Early design concept of the shell lander with a full enclosure

The here proposed half-shell concept has some advantages. The overall mass and volume is reduced as less crushable elements are needed. The interface to both the mobile lander as well as to the mother spacecraft is less complex. The risk of possible jammed levers or hinges by the impact is removed. And due to the directed descent the impact vector is vertical to the surface requiring mainly uni-axial deformable energy absorption, which matches best the honeycomb core crush performance.

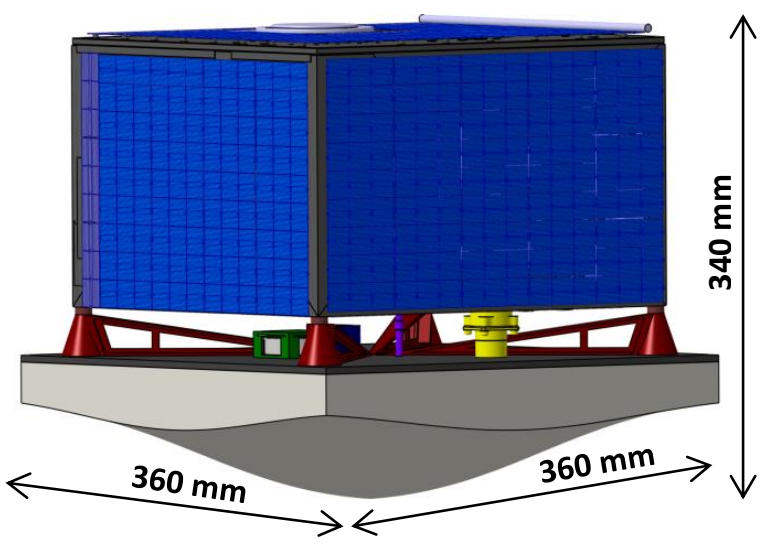

Fig. 14: GSL concept including a generic mobile instrument carrier attached to the ECAP.

Fig. 14 shows the overall size of the GSL which is $360 \times 360 \times 340 \mathrm{~mm}^{3}$, while the CHS has a maximum height of $100 \mathrm{~mm}$. The width of the CHS is driven by an attitude tolerance of $5^{\circ}$. It is therefore slightly larger than the attached mobile lander to avoid unwanted side contacts. The shape and curvature of the CHS is defined by the touchdown conditions which can range from a flat surface to an impact on an obstacle. From this it follows that the impact does not necessarily go through the Center of Gravity (CoG) as can be seen in Fig. 15.
Equation (19) defines the mass distribution for the lander with respect to the distance from its CoG.

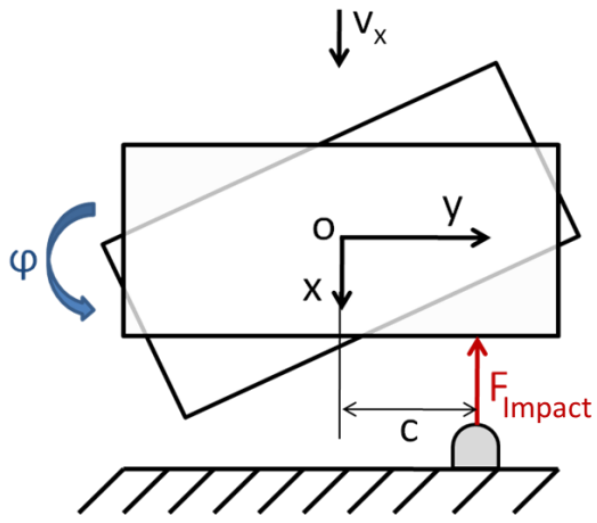

Fig. 15: Scheme for calculating the mass distribution when impacting on an obstacle

$$
m(c)=\frac{1}{\frac{c^{2}}{J_{\text {Lander }}}+\frac{1}{m_{\text {Lander }}}}
$$

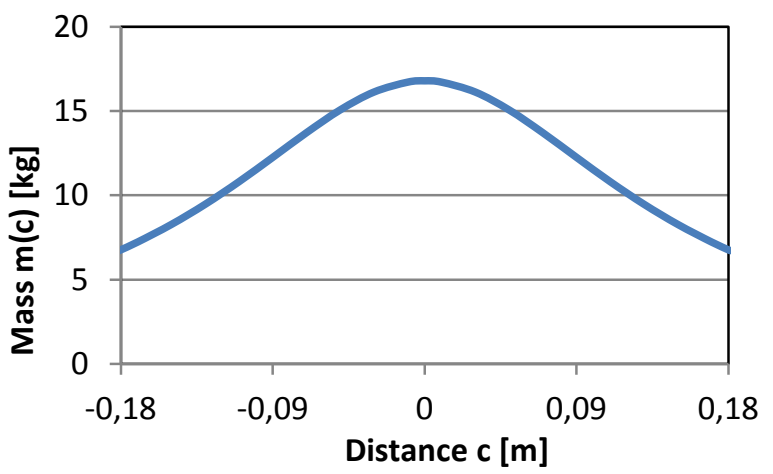

Fig. 16: Calculated mass distribution of the CHS

As a result, it can be seen in Fig. 16 that the full mass is only acting for a central impact. If the lander hits an obstacle at the edge of the CHS, only $40 \%$ of the mass is affecting the crash performance. Consequently, mass optimization is achieved by giving the CHS a curvature as defined by equation (19). Further details can be reviewed in [37] and [38]. Furthermore, it is assumed that any equipment required to implement and to support the landing strategy and which are not used anymore after landing shall be ejected after the CHS has been spent. The flywheel and additional instrumentation is therefore mounted on the ECAP. Instrumentation are sensors which acquire data on the CHS performance such as additional accelerometer and displacement sensors. In that regard this data would support analysis to distinct between soil mechanical observations upon impact and soil contribution to energy absorption as 
opportunity science. Such sensors are marked as optional in the mass budget.

The vertical cross beams and the supporting electronics are mounted to the sandwich-plate on top of the CHS (Fig. 17) and the required power supply is provided by the mobile carrier via the separable umbilical connector. This connector as well as the NEA are both MASCOT heritage and have been flown successfully on the Hayabusa2 mission (Fig. 18). The proposed flywheel is a standard cubesat component. Commercial examples are presented in Fig. 19.

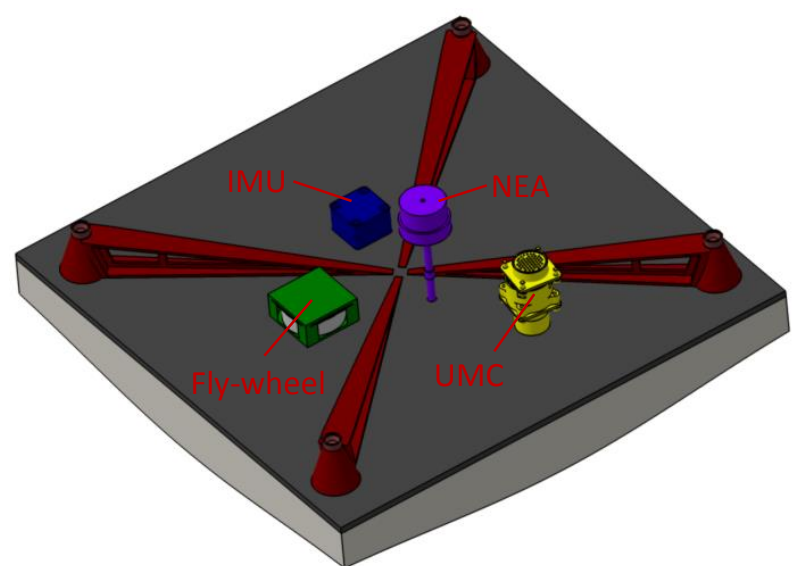

Fig. 17: ECAP platform with its lander interface frame (red) and supporting electronics
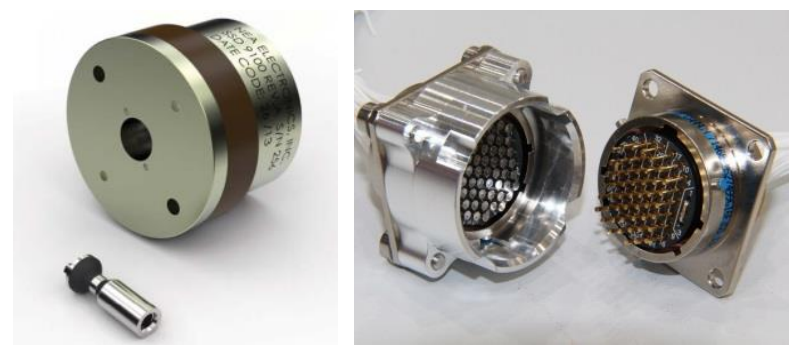

Fig. 18: Separation electronics as used in the Hayabusa2/MASCOT mission. Left: NEA model 9100 (image credit NEA Electronics). Right: Umbilical separation connector (UMC).
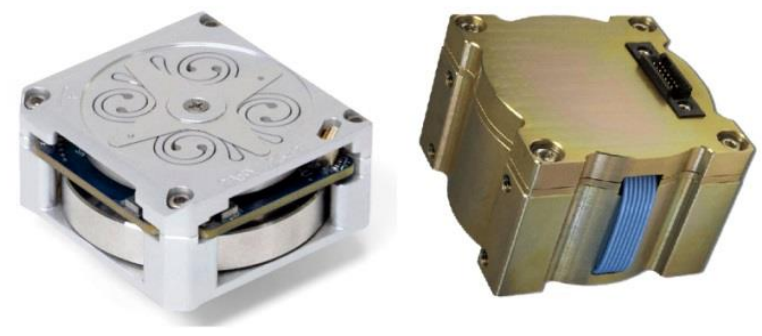

Fig. 19: Commercial flywheels. Left: MicroWheel (RWP015) from Blue Cayon Technologies. Right: CubeWheel Medium from CubeSatShop.com

\subsection{Compatibility to other Mobile Landers}

The ECAP design is flexible and its underlying concept intended to support the landing of mobile surface elements in general. In a "What-If"-study we briefly assess the application also to the JPL Hedgehog and the APL POGO. The interface design can be adapted to hold either of these landers. A comparing sketch is given in Fig. 20. Each type of lander would need to be equipped with a NEA or similar hold down and release mechanism (HDRM) and requires the capability to send a high but very short impulse current (e.g. via the internal battery) in order to activate the release. For the MASCOT-2 and POGO landers a separable umbilical connector would provide power supply for the fly-wheel as well as data interface for any attached sensors. The Hedgehog lander on the other hand has the advantage, that it can make use of its already integrated tri-axial momentum wheels which are used primarily for locomotion across the surface. Here, no additional fly-wheel or connector is required reducing the total mass of the shell platform. The resulting system mass, including the addition of a spacecraft interface and push-off mechanism for initial deployment is presented in Table 3.

Table 3: Mass breakdown of 3 different types of mobile landers equipped with the ECAP and a $\mathrm{S} / \mathrm{C}$ interface and push-off mechanism.

\begin{tabular}{cccc}
\hline & $\begin{array}{c}\text { APL } \\
\text { POGO }\end{array}$ & $\begin{array}{c}\text { DLR } \\
\text { MASCOT-2 }\end{array}$ & $\begin{array}{c}\text { JPL } \\
\text { Hedgehog }\end{array}$ \\
\hline Lander mass & $10 \mathrm{~kg}$ & $13.8 \mathrm{~kg}$ & $25 \mathrm{~kg}$ \\
\hline $\begin{array}{c}\text { S/C Interface } \\
\text { and Push-off. }\end{array}$ & $1,5 \mathrm{~kg}$ & $1,5 \mathrm{~kg}$ & $1,8 \mathrm{~kg}$ \\
\hline $\begin{array}{c}\text { ECAP } \\
\text { platform }\end{array}$ & $1,5 \mathrm{~kg}$ & $1,5 \mathrm{~kg}$ & $1,2 \mathrm{~kg}$ \\
\hline Total & $13 \mathrm{~kg}$ & $16,8 \mathrm{~kg}$ & $28 \mathrm{~kg}$ \\
\hline
\end{tabular}



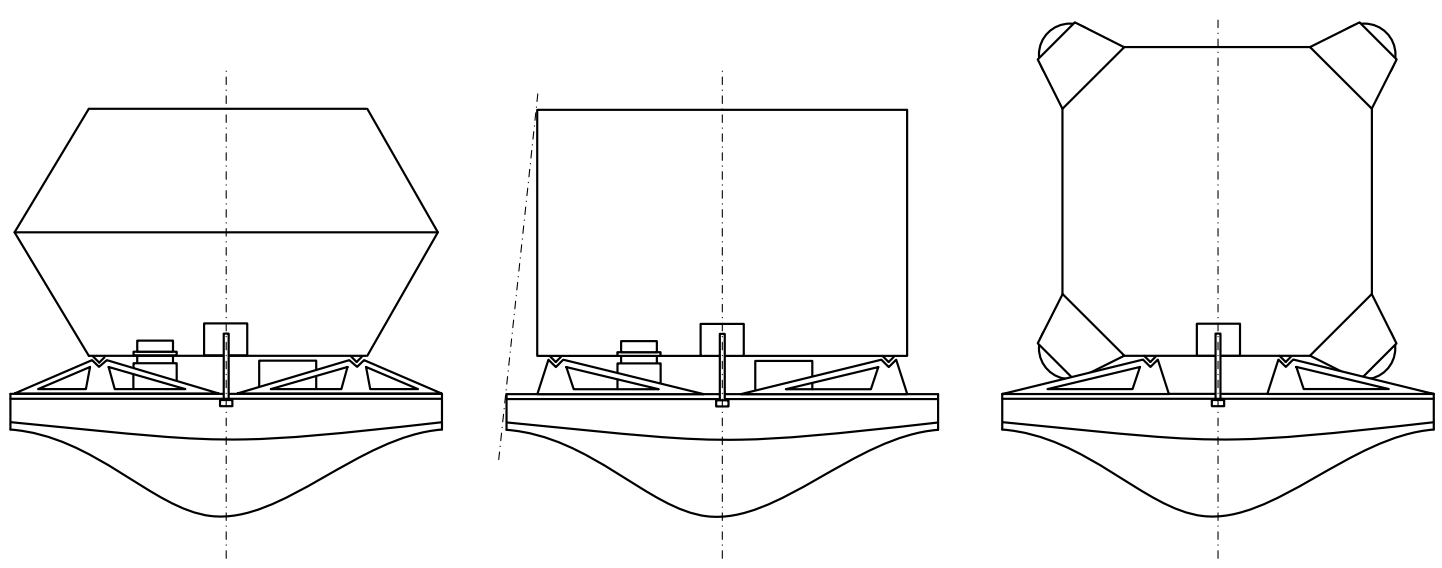

Fig. 20: Comparing sketch (to scale) showing the ECAP attached to various mobile landers, including from left to right the APL POGO, the DLR MASCOT-2 and the JPL Hedgehog

\section{Proof of Concept Testing}

In order to validate the shell lander concept and to improve the accuracy of our FE analysis we have performed laboratory impact tests at the landing and mobility test facility (LAMA) at DLR Bremen, which is normally used to study and test the landing events of larger landers for Moon and Mars [39][40]. The touchdown speed is determined foremost by the kinetic energy (see section 4) which makes it easy to test on Earth $\left(\mathrm{g}=9.81 \mathrm{~m} / \mathrm{s}^{2}\right)$ without the need for $\mathrm{a}$ microgravity environment. One way to test would be to simply drop a test object from a certain height to achieve the desired impact speed. For example, the equivalent drop height for an impact speed of $3 \mathrm{~m} / \mathrm{s}$, using equation (8) would be $46 \mathrm{~cm}$. The force vector due to gravity is in this case always vertical.

$$
\overrightarrow{\mathrm{F}_{\mathrm{G}}}=-\mathrm{mg}
$$

Another way to test is to use a pendulum, which is deflected from its vertical rest position to the same height to achieve the same impact speed (Fig. 21).

$$
h=L(1-\cos \varphi)
$$

The resulting force vector in this case is then a function of deflection angle $\varphi$.

$$
\overrightarrow{\mathrm{F}_{\mathrm{R}}}=-\mathrm{mg} \cos \varphi
$$

The advantage of using a pendulum is that during the moment of impact $(\varphi=0)$,

$$
\overrightarrow{\mathrm{F}_{\mathrm{G}}}=\overrightarrow{\mathrm{F}_{\mathrm{P}}}=-\mathrm{mg} \sin \varphi
$$

becomes 0 and $F_{R}$ is perpendicular to the gravity vector. The test object is therefore decoupled from gravity and thus reacts only due to the resisting force of the obstacle. In this respect, one can analyze the bouncing effect of the test object when being deflected off the ground or obstacle. The bouncing ratio or coefficient of restitution $(\mathrm{COR})$ is then determine by

$$
e=\frac{v_{\text {out }}}{v_{\text {in }}}
$$

where $v_{\text {in }}$ is the impact velocity just before contact and $v_{\text {out }}$ is the deflected velocity just after impact in the opposite direction. This is important when focusing on landing on small bodies with low gravities as this parameter defines for example how big the landing error ellipse will be and how long it takes for the lander to settle on the surface.

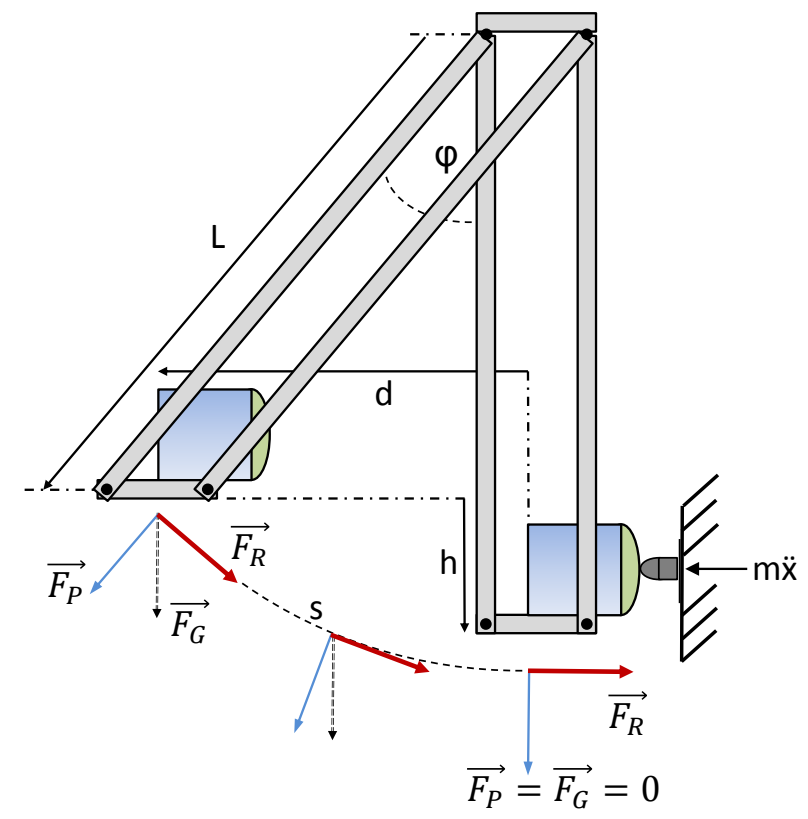

Fig. 21: Rigid parallel pendulum - schematic 


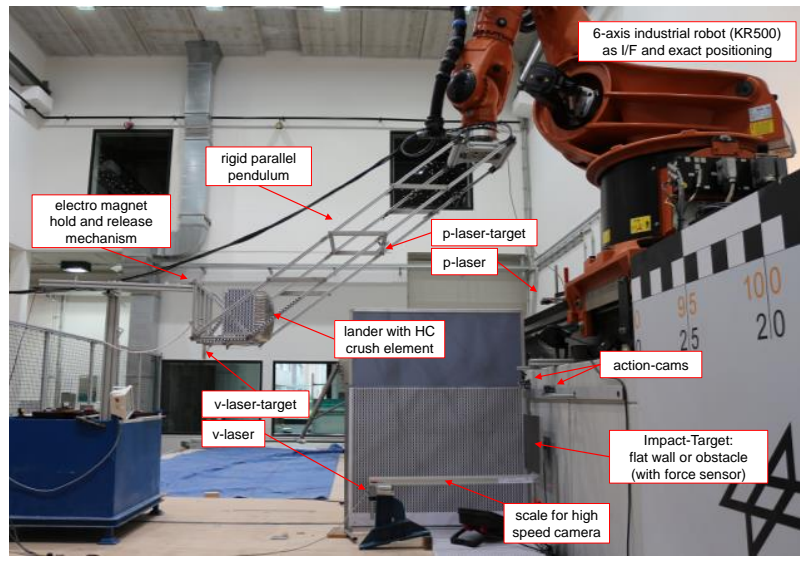

Fig. 22: Pendulum test setup for the shell lander impact tests

As it is shown in Fig. 21 and Fig. 22, the pendulum setup is made of a rigid aluminum frame forming a parallel pendulum. This technique is used to ensure that the test object is always horizontal and in line with the impact target. In addition, a rigid $\mathrm{Al}$-frame is preferred over ropes or wires to analyze and to account for induced vibrations to the pendulum during the impact. The setup included a load cell directly behind the impact target, tri-axial accelerometers on the test object, laser range finders as well as high-speed and video cameras to characterize the impact event as well as the pendulum itself. Tests were performed for the expected worst cases,

A. Flat: impacting on a hard flat surface representing bare rock or a boulder much larger the size of the lander (Fig. 23, top),

B. Penetrator: impacting on a hard obstacle representing a boulder much smaller the size of the lander (Fig. 23, bottom),

Beside the crush performance of the AL-honeycomb core $(\sigma=0.1725 \mathrm{MPa})$, further objectives were to investigate the influence of the face sheet laminates (layers tested: 0, 1 and 2 layers of a Dyneema fabric) as well as the dependence of the impact velocity (velocities tested: 2,3 and $4 \mathrm{~m} / \mathrm{s}$, respectively). Exemplary results can be seen below.

Fig. 24 - Fig. 31 show impact cases A and B for a crash-pad without a face sheet laminate. For a flat impact the contact area A (see equation (2)) increases quickly due to the curvature of the crash-pad. Consequently, the resistance $\mathrm{F}_{\text {cr }}$ provided by the target plate is large and the deceleration time and distance $\mathrm{s}$ is small. Due to this, the impact force and acceleration are large as well. For a penetrator impact without a face sheet the contact area and therefore the resistance is small and remains constant due to the small and constant cross section. For lower velocities (2 and 3 $\mathrm{m} / \mathrm{s}$ ) the crash-pad was able to prevent the impactor to protrude towards the lander. For higher velocities (e.g. 4 $\mathrm{m} / \mathrm{s}$ ) the crash-pad was not able to stop the impactor from breaking through.

Fig. 32 - Fig. 39 shows impact cases A and B for a crash-pad including a face sheet laminate. Similar to the case without a face-sheet, the contact area for a flat impact increases quickly due to the curvature of the crash-pad. Consequently, the resistance provided by the target plate is large and the deceleration time and distance is small. Similarly, the impact force and acceleration are large as well. However, when comparing now the impact case for a penetrator including a face sheet, the contact area increases also, but much slower than for the flat case. Due to this, the impact force and acceleration are higher than for the non-laminate case, but much lower as compared to the flat impact cases since the crash-pad is given more time to spread the impact force via the face sheet over a wider area. Even for high velocity impacts, the face sheet does effectively prevent the impactor to break through. However, the amount of energy being absorbed is higher in the flat impact case compared to the penetrator impact case (see Fig. 41 and Fig. 42). These results match closely the test predictions acquired by FE analysis prior to these tests (see Fig. 23).
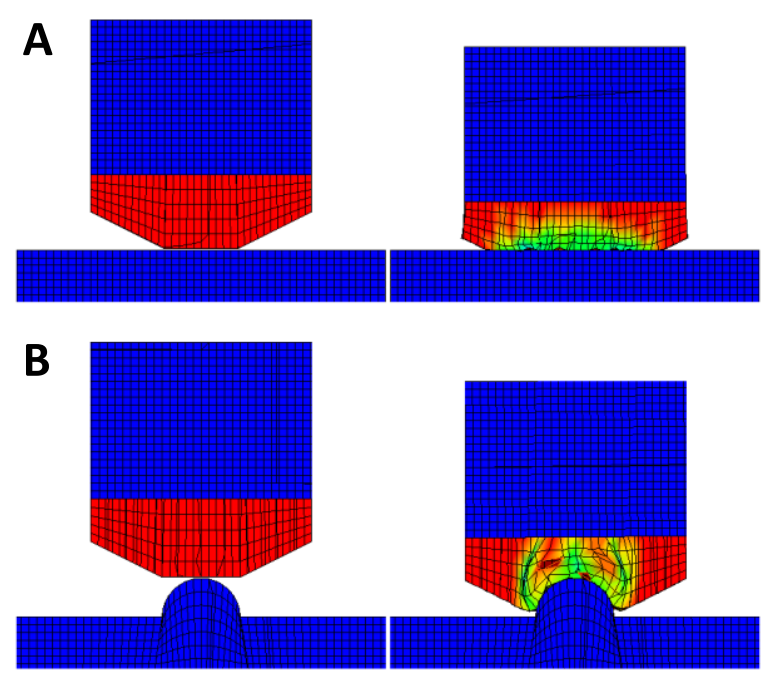

Fig. 23: FE simulation of the CHS (red) attached to a generic lander (blue): A) impact on a flat surface; B) impact on an obstacle

As mentioned before, impact cases $\mathrm{A}$ and $\mathrm{B}$ present worst cases on opposite ends. Flat impacts result in very high acceleration values and penetrator impact cases present a high risk of breaking through the protective shield. They act therefore as boundary condition to which a crash-pad has to be designed to. The face sheet, on the other hand brings these boundaries closer together. With the right combination of resisting core material and face sheet laminate a crash-pad can be designed to ensure both, the capability to protect the 
lander from structural damage as well as to limit the residual shock acceleration for sensitive instruments onboard.

Summarizing results are presented in (Fig. 40 - Fig. 42). Blue squares mark flat impact cases and red triangles mark penetrator impact cases. Test object identifiers describe larger square pads (T) and smaller rectangular pads $(\mathrm{S})$. Indices $0-2$ indicate the number of face sheets used. Fig. 40 shows the peak acceleration over varying impact speeds. As mentioned above, for penetrator cases with no-laminate the shock acceleration remains constant as for all other cases the acceleration increases linear with impact speed. Highest values result for flat impacts, lowest values result from penetrator cases. Fig. 41 presents the crush energy (absorbed energy during impact) for varying impact speeds.
From the linear trend of all cases it follows that the value of crush energy, which is also the indicator for the crash-pads performance, is directly and linear dependent from the impact speed. The higher the impact speed and consequently the higher the impact energy, the more energy is absorbed. This means also, that the crush energy is independent from the impact energy. Fig. 42 shows that $75-85 \%$ of the kinetic impact energy is absorbed within the crash-pad depending only on the used material combination. In terms of the aforementioned COR, and using face sheet material, these relates to an $e$ of $0.4-0.5$. This can be reduced further, however, using softer core materials which would also lower peak acceleration values specifically for flat impact cases.

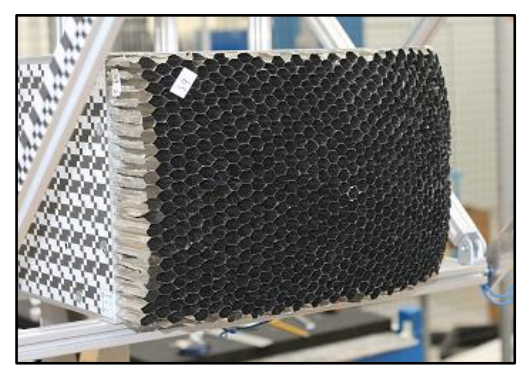

Fig. 24: Crash-pad w/o a face sheet before test

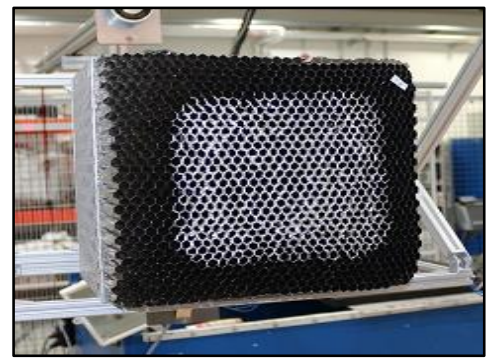

Fig. 26: Crash-pad w/o a face sheet after impact with a hard flat surface $(v=4 \mathrm{~m} / \mathrm{s})$

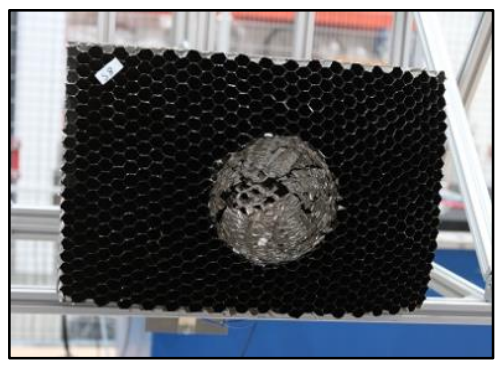

Fig. 29: Crash-pad w/o a face sheet after impact with a hard obstacle $(v=3 \mathrm{~m} / \mathrm{s})$
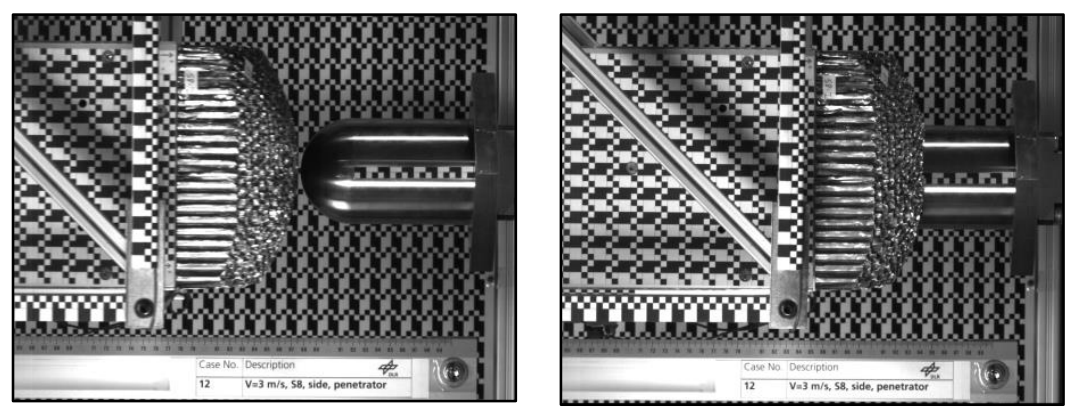

Fig. 25: Test example: penetrator impact w/o face sheet laminate $(v=3 \mathrm{~m} / \mathrm{s})$ : before impact (left), after impact (right)

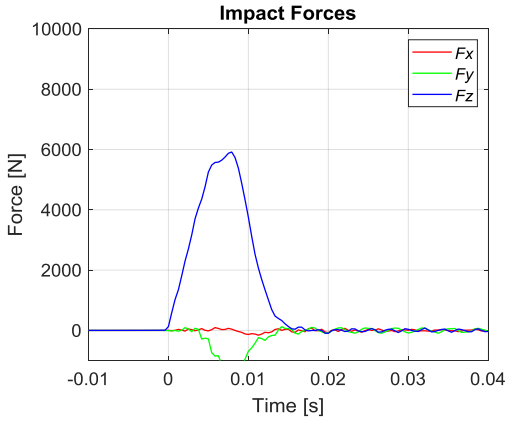

Fig. 27: Impact force, flat impact w/o face sheet $(v=4 \mathrm{~m} / \mathrm{s})$

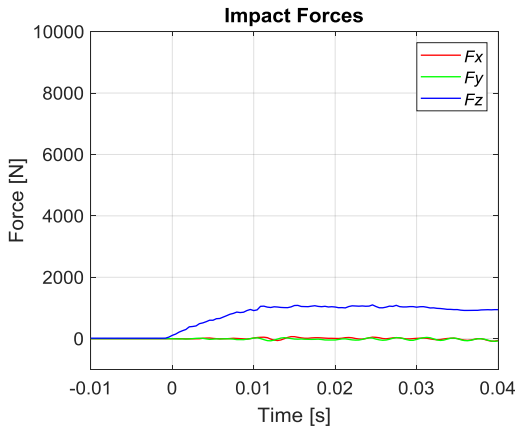

Fig. 30: Impact force, boulder impact w/o face sheet $(v=3 \mathrm{~m} / \mathrm{s})$

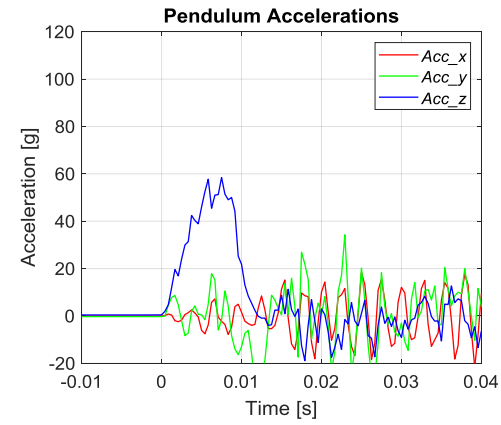

Fig. 28: Impact acceleration, flat impact w/o face sheet $(v=4 \mathrm{~m} / \mathrm{s})$

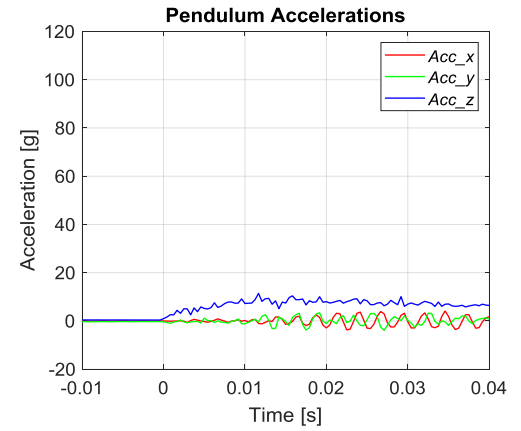
impact w/o face sheet $(\mathrm{v}=3 \mathrm{~m} / \mathrm{s})$
Fig. 31: Impact acceleration, boulder 


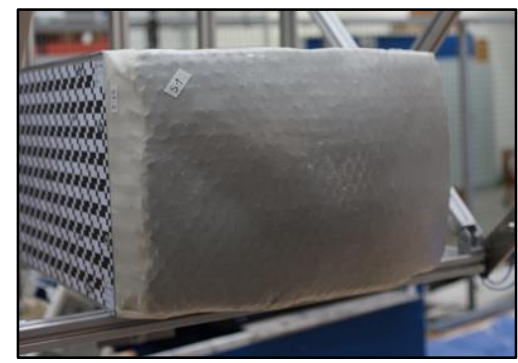

Fig. 32: Crash-pad with a face sheet laminate before test

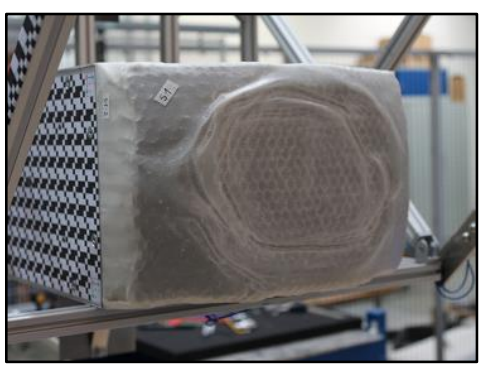

Fig. 34: Crash-pad with face sheet after impact with a hard flat surface $(v=4 \mathrm{~m} / \mathrm{s})$

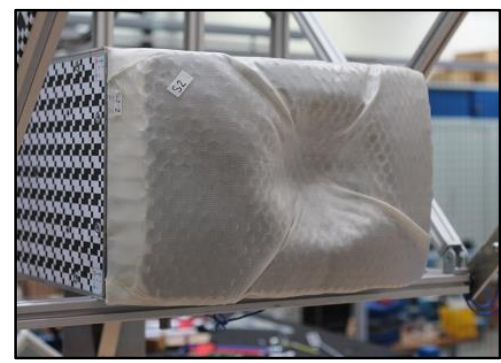

Fig. 37: Crash-pad with face sheet after impact with a hard obstacle $(\mathrm{v}=4 \mathrm{~m} / \mathrm{s})$

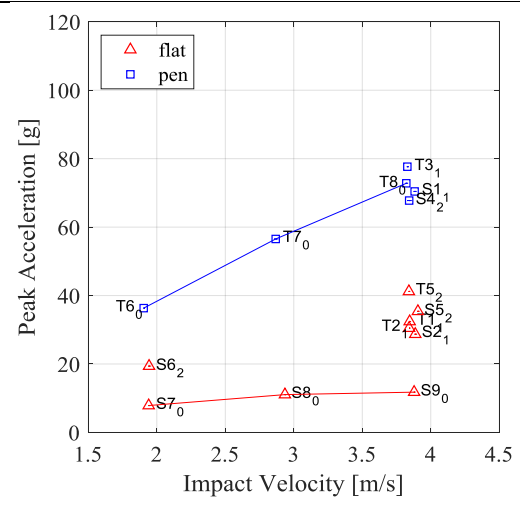

Fig. 40: Peak acceleration wrt impact velocity
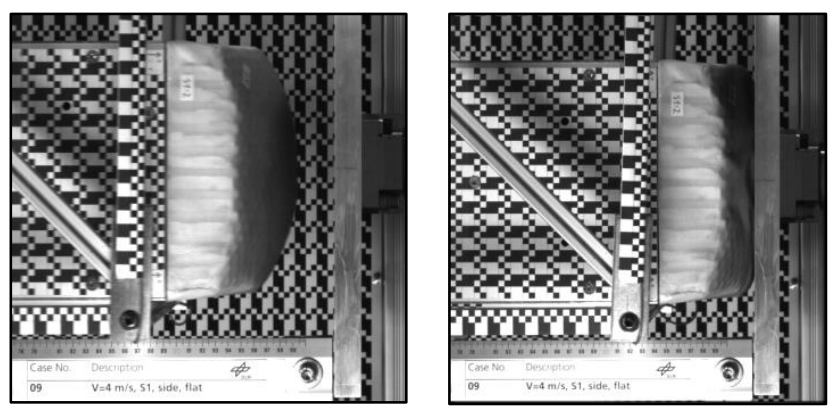

Fig. 33: Test example: flat impact with a face sheet laminate $(v=4 \mathrm{~m} / \mathrm{s})$ : before impact (left), after impact (right)

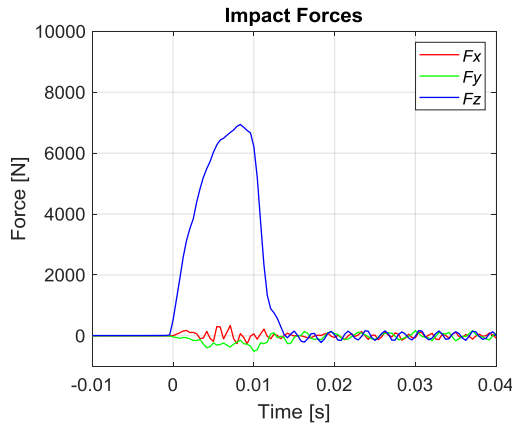

Fig. 35: Impact force, flat impact with face sheet $(\mathrm{v}=\mathbf{4} \mathrm{m} / \mathrm{s})$

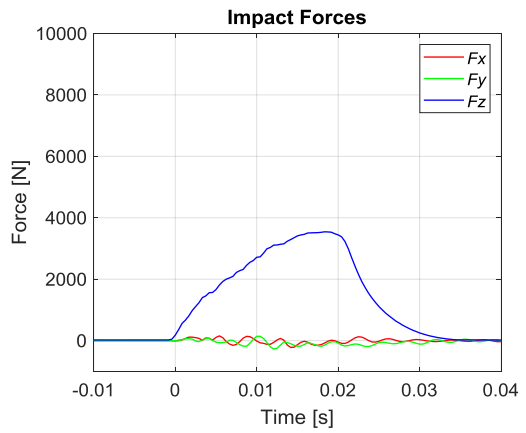

Fig. 38: Impact force, boulder impact with face sheet $(v=4 \mathrm{~m} / \mathrm{s})$

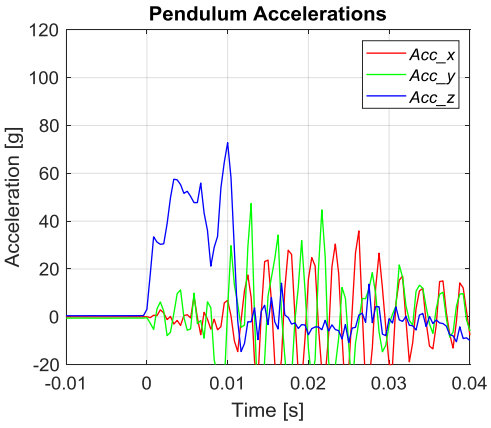

Fig. 36: Impact acceleration, flat impact with face sheet $(v=4 \mathrm{~m} / \mathrm{s})$

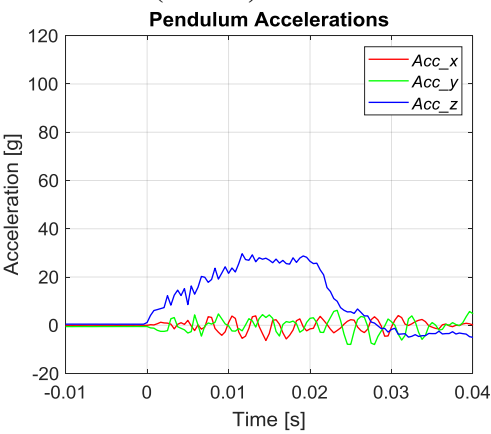

Fig. 39: Impact acceleration, boulder impact with face sheet $(\mathrm{v}=4 \mathrm{~m} / \mathrm{s})$

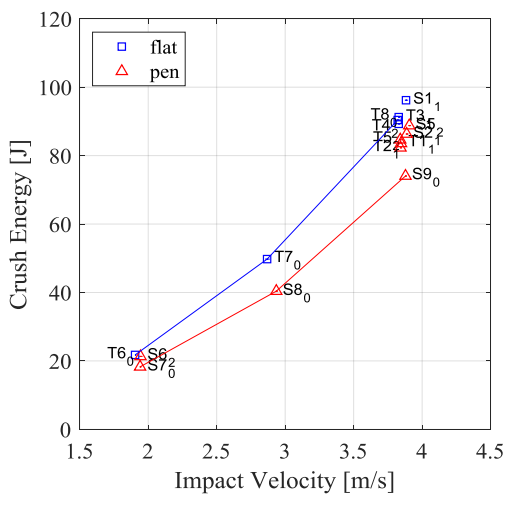

Fig. 41: Crush energy wrt impact velocity

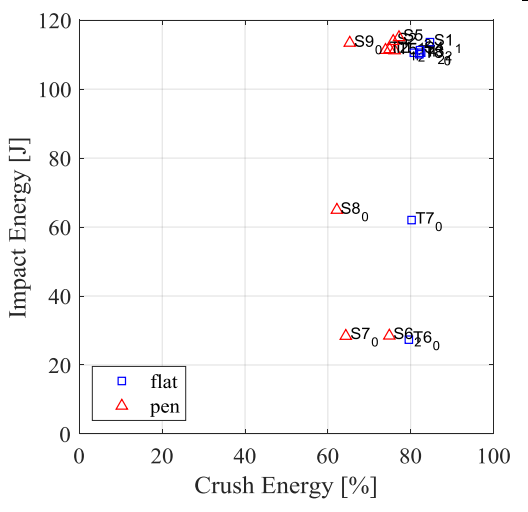

Fig. 42: Relation between impact energy and crush energy 
$69^{\text {th }}$ International Astronautical Congress (IAC), Bremen, Germany, 1-5 October 2018. Copyright 2018 by German Aerospace Center (DLR), Bremen, Germany. Published by the IAF, with permission and released to the IAF to publish in all forms.

\section{Conclusions}

Small carry-on landers have proven to be valuable assets by avoiding additional complexity of the main satellite and transferring the risk (and cost) of close surface maneuvers entirely or at least to some extend to an independent deployable system. In order to enable the exploration and landing on medium-size airless bodies between diameters of $10-50 \mathrm{~km}$ we present in this paper the idea of advancing carry-on landers with a crushable-shell protection system to sustain higher landing velocities in the range of $1-5 \mathrm{~m} / \mathrm{s}$. For this, we first classified carry-on landers with respect to their touchdown and operational strategy to find similarities and identify key technologies. We evaluated the constraints of ballistic deployments for different target bodies as well as identified the niche for using simple honeycomb impact dampers compared to optional retropropulsion systems. Further, we introduced the system design of a "guided" Shell Lander using a generic instrument carrier attached to a single ejectable crashpad with stabilizing capability to protect the instrument carrier from structural damage, limit internal shock loads for sensitive payloads as well as reduce the amount of bounces on the surface. Finally, we presented a mission architecture for a reference case to the Martian moon Phobos as well as provided a proof of concept based on laboratory impact tests.

\section{References}

[1] Biele, J., Ulamec, S., Maibaum, M., et al., The landing $(s)$ of Philae and inferences about comet surface mechanical properties, Science 349(6247) aaa9816, 2015

[2] Ho, T.-M., Baturkin, V., Grimm, C.D., et al., MASCOT - The Mobile Asteroid Surface Scout Onboard the Hayabusa2 Mission, Space Science Reviews 208(1) 339-374, 2017

[3] Michel, P., Cheng, A., Küppers, M., et al, Science case for the Asteroid Impact Mission (AIM): A component of the Asteroid Impact \& Deflection Assessment (AIDA) mission, Advances in Space Research 57(12) 2529-2547, 2016

[4] Michel, P., Cheng, A., Küppers, M., et al, The HERA Mission: European Component of the Asteroid Impact and Deflection Assessment (AIDA) Mission to a Binary Asteroid, 49th Lunar and Planetary Science Conference 2018 (LPI Contrib. No. 2083)

[5] NASA Space Science Data Coordinated Archive (NSSDCA), NASA's Discovery Program,

https://nssdc.gsfc.nasa.gov/planetary/discovery. $\underline{\mathrm{html}}$, (accessed 16.05.2018).
[6] NASA Space Science Data Coordinated Archive (NSSDCA), NASA Science Missions, https://science.nasa.gov/missionspage?field division tid $=81 \&$ field phase $t$ tid $=\mathrm{A}$ 11\&=Apply, (accessed 15.09.2018).

[7] Beshore, E., Lauretta, D., Boynton, W., et al., The OSIRIS-REx asteroid sample return mission. In: 2015 IEEE Aerospace Conference pp. 1-14. IEEE.

[8] Kane, V., Proposals to Explore the Solar System's Smallest Worlds, 27 July 2015, http://www.planetary.org/blogs/guest-

blogs/van-kane/20150727-proposals-toexplore-the-solar-systems-smallestworlds.html, (accessed 09.2018).

[9] Staehle, R. L., Blaney, D., Hemmati, H., et al., Interplanetary CubeSats: Opening the Solar System to a Broad Community at Lower Cost, Journal of small satellites: research, technology, and systems, 2(1) 161-186, 2013

[10] Lange, C., Ho, T., Grimm, C., et al., Nanoscale landers and instrument carriers: enhancing larger mission's science return by investing in low cost solutions: the MASCOT- 1 to $X$ and ROBEX examples, In: 11th Low Cost Planetary Missions Conference, Berlin, Germany, 2015

[11] Adams, E., Hohlfeld, E., Hill, S., et al., Asteroid in situ exploration using planetary object geophysical observer (POGO), In: 2016 IEEE Aerospace Conference, pp 1-10,

[12] Reid, R. G., Roveda, L., Nesnas, I. AD, et al., Contact dynamics of internally-actuated platforms for the exploration of small solar system bodies, In: Proceedings of i-SAIRAS, pp.1-9, Montreal, Canada, 2014

[13] Hockman, B. J., Frick, A., Reid, R. G. et al., Design, Control, and Experimentation of Internally-Actuated Rovers for the Exploration of Low-gravity Planetary Bodies, Journal of Field Robotics 34(1) 5-24, 2017

[14] Ho, T., Lange, C., Grimm, C., et al., A Mobile Asteroid Surface Scout for the AIDA Mission, In. EGU General Assembly, vol.18 p.16163, Vienna, Austria, 2016

[15] Ho, T., Biele, J., Lange, C., et al., AIM MASCOT-2 Asteroid Lander Concept Design, 20 February 2016, ESA General Studies Programme, https://gsp.esa.int/gsp-study-view//wcl/gaUaMHco1QJ9/10192/aim-mascot-2asteroid-lander-concept-design, (accessed 15.09.2018).

[16] ESA, Small Planetary Platforms (SPP) Executive Summary, CDF Study Report, CDF178(C), January 2018

[17] Ball, A., Garry, J., Lorenz, R., Kerzhanovich, V., Planetary landers and entry probes, 
$69^{\text {th }}$ International Astronautical Congress (IAC), Bremen, Germany, 1-5 October 2018. Copyright 2018 by German Aerospace Center (DLR), Bremen, Germany. Published by the IAF, with permission and released to the IAF to publish in all forms.

Cambridge University Press, 2007

[18] Yano, H., Kubota, T., Miyamoto, H., et al., Touchdown of the Hayabusa spacecraft at the Muses Sea on Itokawa, Science 312(5778) 1350-1353, 2006

[19] Yoshimitsua, T., Kubota T., Nakatani I., et al., Micro-hoppingrobot for asteroid exploration, Acta Astronautica 52(2-6) 441-446, 2003

[20] NASA Space Science Data Coordinated Archive (NSSDCA), Phobos-Grunt, NSSDCA/ COSPAR ID: 2011-065A, https://nssdc.gsfc.nasa.gov/nmc/spacecraftDispl ay.do?id=2011-065A, (accessed 07.05.2018).

[21] Watanabe, S., Tsuda, Y., Yoshikawa, M., et al., Hayabusa2 Mission Overview, Space Science Reviews 208(1) 3-16, 2017

[22] NASA Space Science Data Coordinated Archive (NSSDCA), OSIRIS-REx, NSSDCA/ COSPAR ID: 2016-055A, https://nssdc.gsfc.nasa.gov/nmc/spacecraftDispl ay.do?id=2016-055A, (accessed 16.05.2018).

[23] JAXA, Martian Moons eXploration (MMX) Mission Overview, www.isas.jaxa.jp/en/topics/files/MMX170412 EN.pdf, (accessed 15.09.2018).

[24] Hockman, B. J, Pavone, M., Stochastic Motion Planning for Hopping Rovers on Small Solar System Bodies, In: Proceedings of ISRR, 2017.

[25] Hockman, B. J., Frick, A., Reid, R. G., et al., Design, Control, and Experimentation of Internally-Actuated Rovers for the Exploration of Low-gravity Planetary Bodies, Journal of Field Robotics, 34(1), 5-24, 2017

[26] Witte, L., Touchdown Dynamics and the Probability of Terrain Related Failure of Planetary Landing Systems, Universität Bremen, 2015

[27] Hexcel Corporation, HexWeb CR III Product Data Sheet, 2017 [www.hexcel.com, accessed 04.09.2018]

[28] Lemmer, K., Propulsion for CubeSats, Acta Astronautica 134, p. 231-243, 2017, doi:10.1016/j.actaastro.2017.01.048

[29] X. Yu and J. Zhou, CubeSat: A candidate for the asteroid exploration in the future, 2014 International Conference on Manipulation, Manufacturing and Measurement on the Nanoscale (3M-NANO), Taipei, 2014, pp. 261 -
265. doi: 10.1109/3M-NANO.2014.7057349

[30] Krasinsky, G. A., Pitjeva, E. V., Vasilyev, M. V., et al., Hidden Mass in the Asteroid Belt, Icarus 158(1) 98-105, 2002

[31] Weissman, P. R., Lowry, S. C., Structure and density of cometary nuclei, Meteoritics \& Planetary Science 43(6) 1033-1047, 2008

[32] Lange, C., Ho, T. M., Grimm, C. D., et al. Exploring Small Bodies: Nanolander andspacecraft options derived from the Mobile Asteroid Surface Scout. Advances in Space Research, 2017

[33] Bibring, J. P., Rosenbauer, H., Boehnhardt, H., et al., The ROSETTA lander ("PHILAE") investigations. Space science reviews, 128(1-4), 205-220, 2007

[34] Back to Mars: 1988, Soviet Robots in the Solar System, November 3, 2015 , http://ourairports.biz/?p=2955, (accessed 15.09.2018).

[35] The Final Soviet Debacle, 05 September 2018 (last update), Bedford Astronomy Club, Global Surveyor, https://www.astronomyclub.xyz/globalsurveyor/the-final-soviet-debacle.html, (accessed 15.09.2018).

[36] Hoggett, R., 1983-7 - PrOP-F Phobos Hopper, 13 December 2012, http://cyberneticzoo.com/walkingmachines/1983-7-prop-f-phobos-hoppersoviet/,

[37] Schröder, S., Grimm, C., Witte, L., A Crushable Shell for Small Body Landers, In: Proceedings of DLRK 2018

[38] Schröder, S., Reinhardt, B., Brauner, C., et al., Development of a Marslander with crushable shock absorber by virtual and experimental testing. Acta Astronautica, 134, 65-74. 2017

[39] Schröder, S., Witte, L., van Zoest, T. Applications for a Gracity Compensation Test Facility for Planetary Surface Mobility, In: Proceedings of DLRK 2011

[40] Schröder, S., Biele, J., Block, J., et al., Philae Landing Test at the Landing and Mobility Test Facility (LAMA). In: Proceedings of the 64th International Astronautical Congress, 2013 